Discrete Comput Geom 35:329-358 (2006)

DOI: $10.1007 / \mathrm{s} 00454-005-1207-\mathrm{x}$

Geometry

\title{
Rhombus Tilings: Decomposition and Space Structure
}

\author{
Frédéric Chavanon ${ }^{1}$ and Éric Rémila ${ }^{1,2}$ \\ ${ }^{1}$ Laboratoire de l'Informatique du Parallélisme, \\ umr 5668 CNRS-INRIA-UCB Lyon 1-ENS Lyon, \\ 46 allée d'Italie, 69364 Lyon cedex 07, France \\ \{Frederic.Chavanon,Eric.Remila\}@ens-lyon.fr \\ ${ }^{2}$ GRIMA, IUT Roanne, \\ 20 avenue de Paris, 42334 Roanne cedex, France
}

\begin{abstract}
We study the spaces of rhombus tilings, i.e. the graphs whose vertices are tilings of a fixed zonotope. Two tilings are linked if one can pass from one to the other by a local transformation, called a flip. We first use a decomposition method to encode rhombus tilings and give a useful characterization for a sequence of bits to encode a tiling. We use the previous coding to get a canonical representation of tilings, and two order structures on the space of tilings. In codimension 2 we prove that the two order structures are equal. In larger codimensions we study the lexicographic case, and get an order regularity result.
\end{abstract}

\section{Introduction}

Rhombus tilings are tilings of zonotopes with rhombohedra. More precisely, we fix a sequence $V=\left(v_{1}, v_{2}, \ldots, v_{D}\right)$ of vectors of $\mathbb{R}^{d}$ (such that each subsequence $\left(v_{i_{1}}, v_{i_{2}}\right.$, $\left.\ldots, v_{i_{d}}\right)$ of length $d$ is a basis of $\left.\mathbb{R}^{d}\right)$ and a sequence $M=\left(m_{1}, m_{2}, \ldots, m_{D}\right)$ of positive integers. The tiled zonotope $Z(V, M)$ is the set $Z(V, M)=\left\{v \in \mathbb{R}^{d}, v=\right.$ $\left.\sum_{i=1}^{D} \lambda_{i} v_{i}, \lambda_{i} \in\left[-m_{i}, m_{i}\right]\right\}$, and each tile is a rhombohedron defined by a set $\left\{v_{i_{1}}, v_{i_{2}}\right.$, $\ldots, v_{i_{d}}$ \} of $d$ vectors of $V$. The notion of flip on tilings is carefully studied. Assume that a tiling $T$ of $Z$ contains $d+1$ tiles which pairwise share a facet. In such a case a new tiling $T_{\text {flip }}$ of $Z$ can be obtained by a flip consisting of changing the position of the $d+1$ previous tiles. The space of tilings of $Z$ is the graph whose vertices are tilings of $Z$ and two tilings are linked by an edge if they differ by a single flip. The structure of spaces of tilings is very interesting, since rhombus tilings appear in physics as a classical model for quasicrystals [16].

In this paper we first use ideas (deletion, contraction, minors) issued from matroid theory [1] to get a decomposition method for tilings (Section 2). We see how to encode 
a tiling by a sequence of small tilings, containing $d+1$ tiles. Informally, this encoding can be seen as a piling of (hyper)cubes, in a similar way as has been done by Thurston [15] for the particular case of tilings with lozenges $(d=2$ and $D=3)$ for any polygon.

The first result of the paper (the reconstruction process in Section 3 ) is a characterization of sequences of small tilings which really encode a (big) tiling. This characterization is local in the sense that it can be checked using a set of conditions, each of them using a bounded number of small tilings. This characterization allows us to confer two canonical order structures ( $\leq_{\text {flip }}$ and $\leq_{\text {set }}$ ) to the space of tilings and to introduce a representation of tilings with some basic elements (points, lines, arrows) (Section 4). This representation is used in the following to understand the structure of spaces of tilings.

The complexity of the space of tilings is strongly related with the codimension $c=$ $D-d$. If $c=0$, obviously, there exists a unique tiling. For $c=1$, it can easily be shown (using the piling of cubes interpretation) that the space of tilings can be directed (by $\leq_{\text {flip }}$ ) so as to get a structure of a distributive lattice (see [2] for details about this folklore result). The main argument allowing the structural result is the fact that $\leq_{\text {flip }}=\leq_{\text {set }}$ in codimension 1.

In Section 5 we carefully study the case of codimension 2 and prove the first important result of the paper: for $c=2$, the two canonical orders are equal (even though the order is not a lattice in codimension 2).

When $c>2$, the orders $\leq_{f l i p}$ and $\leq_{\text {set }}$ are not equal and the situation becomes very complex since there exist non-isomorphic spaces of tilings for the same pair $(c, d)$ (for $d>2$ ). In Section 6 we study a particular case of the space of tilings (linked to a particular configuration of vectors), when $c>2$, that we have called the lexicographic case. However, in this case we obtain a strong regularity result about the maximal chains of $\leq_{\text {flip }}$.

As a consequence of our results, the space of tilings can be directed so as to get a graded poset (with single maximal and minimal elements). This result clearly induces the connectivity of the space, which is a non-trivial result. As another consequence, we obtain that flips induce a Markov chain on the space of tilings, whose limit distribution is uniform.

Before this paper, a parallel study had been done by Ziegler [19] about higher Bruhat orders. These are combinatorial structures which can be interpreted as extensions of some specific matroids. We recall that the Bohne-Dress theorem [14] claims that tilings of zonotopes can also be interpreted as extensions of matroids. Thus, the study by Ziegler can be seen as the study of tilings of unitary zonotopes constructed from vectors in a cyclic arrangement (thus higher Bruhat orders are a special case of our lexicographic case). For those zonotopes, Ziegler proved that the space of tilings can be directed so as to get a graded poset (with a unique minimal element and a unique maximal element). Ziegler also gives a counterexample for the equality of orders in dimension 3 with eight vectors. In the opposite direction, Felsner and Weil [9] prove the equality of orders, for $d \leq 2$. To our knowledge, the connectivity problem is still open for other kinds of zonotopes. We mention that Kenyon [11] has proved the connectivity in dimension 2, for spaces of tilings of any polygon.

We have chosen to make an exposition which is accessible for people who have no knowledge of oriented matroid theory. This gives some longer proofs, but difficulties are not hidden in strong theorems about matroids. Nevertheless, since tilings are strongly 
related to oriented matroids by the Bohne-Dress theorem [14], we have also given the matroid interpretation of the material presented. We have also tried to give precise references in [1], the most classical book about oriented matroids.

\section{Tilings of Zonotopes and Minors}

In this paper we deal with a particular case of tilings in $\mathbb{R}^{d}$, called zonotopal rhombus tilings (or tight zonotopal tilings). We now define the fundamental elements studied in the following.

The canonical basis of $\mathbb{R}^{d}$ will be denoted by $\left(e_{1}, e_{2}, \ldots, e_{d}\right)$. Let $V=\left(v_{1}, \ldots, v_{D}\right)$ be a sequence of $D$ vectors in $\mathbb{R}^{d}$ such that $D \geq d$ and each subsequence $\left(v_{i_{1}}, v_{i_{2}}, \ldots, v_{i_{d}}\right)$ is a basis of $\mathbb{R}^{d}$. The parameter $c=D-d$ is called the codimension.

Let $M=\left(m_{1}, \ldots, m_{D}\right)$ be a sequence of $D$ non-negative integers. $m_{i}$ is associated with the vector $v_{i}$ and is called the multiplicity of $v_{i}$. The zonotope $Z(V, M)$ associated with the pair $(V, M)$ is the region of $\mathbb{R}^{d}$ defined by $\left\{v \in \mathbb{R}^{d}, v=\sum_{i=1}^{D} \lambda_{i} v_{i}, \lambda_{i} \in\right.$ $\left.\left[-m_{i}, m_{i}\right]\right\}$. Thus, $Z(V, M)$ is the convex hull of the finite set $\left\{v \in \mathbb{R}^{d}\right.$, $\left.v=\sum_{i=1}^{D} \lambda_{i} m_{i} v_{i}, \lambda_{i} \in\{-1,1\}\right\}$.

One can define classically (see, for example, pp. 51-52 of [18]) the faces, vertices (faces of dimension 0), edges (faces of dimension 1), and facets (faces of dimension $d-1)$ of the zonotope $Z(V, M)$, as it is defined for the convex hull of any finite set. The number $s=\sum_{i=1}^{D} m_{i}$ is the size of the zonotope $Z(V, M)$; we say that $Z(V, M)$ is an $s$-zonotope. The zonotope $Z(V, M)$ is said to be unitary if all the multiplicities are equal to 1 (see Fig. 1 for examples).

Let $Z(V, M)$ be a zonotope. The sequence $V$ of vectors is called the type of $Z(V, M)$. A prototile of $V$ is a unitary zonotope constructed with a subsequence $V^{\prime}$ of $d$ distinct vectors taken in $V$. A sequence $V$ of $D$ vectors of $\mathbb{R}^{d}$ induces $\left(\begin{array}{l}D \\ d\end{array}\right)$ different prototiles. A tile $t$ is a translated prototile, i.e. it is defined by a pair $(p, w)$, where $p$ is a prototile and $w$ is a translation vector (formally, we have $t=w+p$ ). Since tiles are some particular polytopes, their vertices, edges and facets are defined as well. The type of tile is the type of the corresponding prototile.

A tiling $T$ of a zonotope $Z(V, M)$ is a set of tiles constructed with vectors in $V$, such that each intersection between tiles is a face of the tiles (i.e. there is no overlap) and the union of tiles is equal to $Z(V, M)$ (i.e. there is no gap). Two tiles are adjacent if they share a whole facet. We say that $Z(V, M)$ is the support of the tiling $T$. If $Z(V, M)$ is an $s$-zonotope, we say that $T$ is an $s$-tiling.
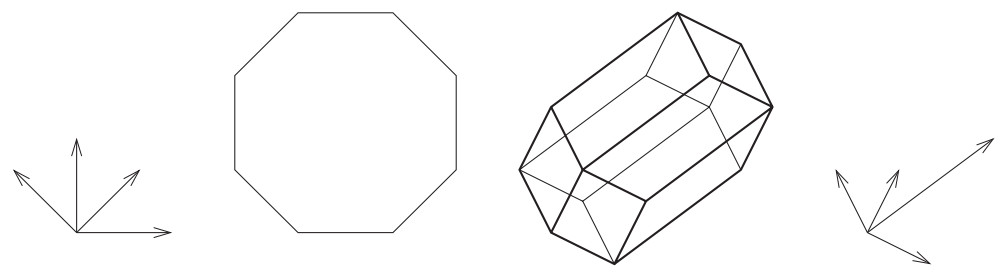

Fig. 1. A two-dimensional zonotope and a three-dimensional zonotope both defined on four vectors. 


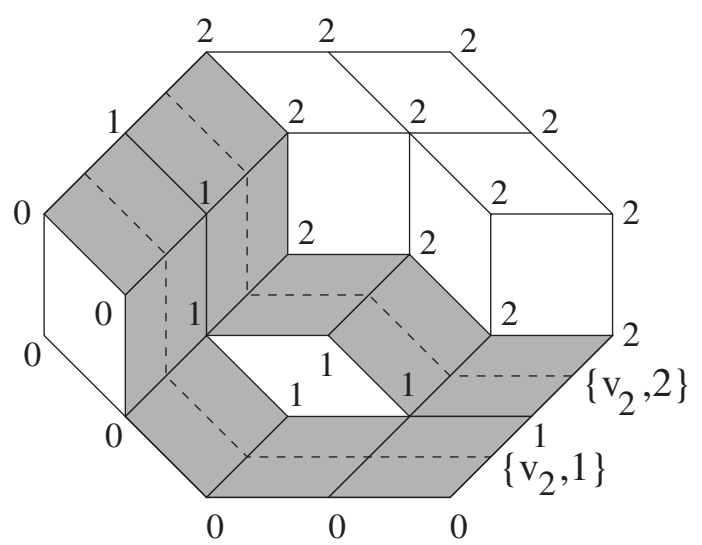

Fig. 2. The 2-located height function and two de Bruijn zones.

\section{1. de Bruijn Zones}

Definition 1 (Lifting, Height Function). Let $Z=(V, M)$ be a zonotope, with $V=$ $\left(v_{1}, \ldots, v_{D}\right)$. A lifting of $V$ is a sequence $U=\left(u_{1}, \ldots, u_{D}\right)$ of vectors of $\mathbb{R}^{d+1}$. For each integer $j$ such that $1 \leq j \leq D$, there exists a real $\alpha_{j}$ such that $u_{j}=\left(v_{j}, \alpha_{j}\right)$.

Let $T$ be a tiling of $Z(V, M)$. An associated lifting is a function $f_{T, U}$ which associates to each vertex of $T_{Z}$ a vector in $\mathbb{R}^{d+1}$ and satisfies the following property: for any pair $\left(x, x^{\prime}\right)$ of vertices of $T$ such that $x^{\prime}=x+2 v_{i}$ and $\left[x, x^{\prime}\right]$ is an edge of $T$, we have $f_{T, U}\left(x^{\prime}\right)=f_{T, U}(x)+2 u_{i}$. See Fig. 2 .

The height function $h_{T, U}$ associated with a lifted tiling $f_{T, U}$, is the component upon $e_{d+1}$ of $f_{T, U}$.

One easily proves that the definition of lifting of a tiling is consistent since a zonotope is homeomorphic to a closed disk of $\mathbb{R}^{d}$.

If $f_{T, U}$ is a given lifting, then each lifting $f_{T, U}^{\prime}$ of $T$ is such that $f_{T, U}^{\prime}=f_{T, U}+k e_{d+1}$, where $k$ denotes a fixed real number. Thus, if $h_{T, U}$ is a given height function, then each height function $h_{T, U}^{\prime}$ of $T$ is such that $h_{T, U}^{\prime}=h_{T, U}+k$. For convention, in this paper the real $k$ is chosen in such a way that the height function does not take negative values and there exists a vertex $v$ such that $h_{T, U}^{\prime}(v)=0$.

Notice that $f_{T, U}$ is defined for the set of vertices of $Z$ and, for each vertex $v$ on the boundary of $Z, f_{T, U}$ does not depend on the tiling $T$ chosen.

The two most used lifting functions are the principal lifting function, defined by $\forall v_{i} \in$ $V, u_{i}=\left(v_{i}, \frac{1}{2}\right)$, and the $k$-located function, where for a fixed integer $k, u_{k}=\left(v_{k}, 1 / 2\right)$ and $\forall i \neq k, u_{i}=\left(v_{i}, 0\right)$. The $k$-located function has the same value on all vertices of a tile whose type does not contain $v_{k}$, and differs by one at the endpoints of an edge of type $v_{k}$. Therefore, the principal function differs by one at the endpoints of each edge of the tiling.

Now, since height functions have been defined, one may introduce the important concept of de Bruijn families and zones, widely used in the core of the paper (see [4] for details). This is the main tool for induction on tilings. 
Definition 2 (de Bruijn Zone, Family). Let $T$ be a tiling of a zonotope $Z(V, M)$, and let $h_{i}$ be the $i$-located function. The de Bruijn family associated with the vector $v_{i}$ is the set of tiles having $v_{i}$ in their type. Moreover, the $j$ th de Bruijn zone is the set of tiles whose $i$-located function is $j-1$ on one facet, and $j$ on the opposite facet. This zone will be denoted $S_{\left\{v_{i}, j\right\}}$ (see Fig. 2).

One sees that a de Bruijn zone $S_{\left\{v_{i}, j\right\}}$ disconnects the tiling into two parts, $T_{\left\{v_{i}, j\right\}}^{+}$and $T_{\left\{v_{i}, j\right\}}^{-}$. The first one is composed of tiles whose vertices have an $i$-located function larger than $j$, and the second corresponds to tiles whose height function is smaller. Hence, for $j<j^{\prime}$, we have $T_{\left\{v_{i}, j\right\}}^{-} \subseteq T_{\left\{v_{i}, j^{\prime}\right\}}^{-}$.

We say that two de Bruijn zones $S_{\left\{v_{i}, j\right\}}$ and $S_{\left\{v_{k}, l\right\}}$ are parallel if $v_{i}=v_{k}$. The intersection of a set of $d$ de Bruijn zones of $T$ which pairwise are not parallel is a tile of $T$. The intersection of a set of $d-1$ de Bruijn zones which pairwise are not parallel is a set of tiles which can be totally ordered in such a way that two consecutive tiles are adjacent. Such an intersection is called a de Bruijn line.

\subsection{Flips}

2.2.1. Tilings of a Unitary $(d+1)$-Zonotope. We first focus on a unitary zonotope of codimension 1 . One easily checks that such a zonotope admits exactly two tilings. Let $V=\left(v_{1}, \ldots, v_{d+1}\right)$ be the sequence of vectors and let $p_{0}$ be the prototile constructed with the $d$ first vectors: there is a tiling $T$ with a tile $t_{0}$ of type $p_{0}$ such that $T_{\left\{v_{d+1}, 1\right\}}^{+}$ is empty and $T_{\left\{v_{d+1}, 1\right\}}^{-}=\left\{t_{0}\right\}$, and one tiling $T^{\prime}$ such that $T_{\left\{v_{d+1}, 1\right\}}^{\prime+}=\left\{t_{0}+v_{d+1}\right\}$ and $T_{\left\{v_{d+1}, 1\right\}}^{\prime-}$ is empty.

Note that $T$ and $T^{\prime}$ are symmetrical. Any pair of tiles of $T$ (or $T^{\prime}$ ) are adjacent, since they form a whole de Bruijn line of $T$. The orders in each de Bruijn line are opposite in $T$ and $T^{\prime}$.

2.2.2. Space of Tilings. Those tilings of a unitary zonotope of codimension 1 can appear, translated, in tilings of a larger zonotope $Z$. Assume that the tiling $T_{z}$ of a unitary $(d+1)$-zonotope $z$ of codimension 1 appears in a tiling $T$ of $Z$, translated by a vector $v$ (i.e. formally, $v+T_{z} \subset T$ ). We say that the tiling $T^{\prime}$ of $Z$, defined by $T^{\prime}=\left(T \backslash\left(v+T_{z}\right)\right) \cup\left(v+T_{z}^{\prime}\right)$, is obtained from $T$ by a geometric flip.

The type of the flip is the type of $z$. It will be denoted by the set of indexes of vectors of its support. We have $\left(\begin{array}{c}D \\ d+1\end{array}\right)$ types of flips; in particular, for $D=d+2$, we have $D$ types of flips.

The space of tilings of a zonotope $Z$ is the symmetric labeled graph whose vertices are the tilings of $Z$, and two tilings are linked by an edge if they differ by a geometric flip. The label of the edge is the type of the corresponding flip.

An important result is that flips induce connectivity between all tilings of zonotopes for $d=2$, i.e. every tiling of a given dimension 2 zonotope $Z$ can be deduced from another tiling of $Z$ by a sequence of flips (see [5], [7], and [11] for details). This is an open question in the case of larger dimensions.

The point now is to study spaces of zonotopal tilings. Despite the fact that rhombic tilings are defined for any dimension, the figures are in dimension 2 for convenience. 


\subsection{Connections with Oriented Matroid Theory}

Tilings are connected with oriented matroids via the Bohne-Dress theorem [14]. In oriented matroids we work with sign vectors, i.e. elements of $\{+,-, 0\}^{n}$, where $n$ is a fixed positive integer. Let $X$ be such a sign vector. For $1 \leq k \leq n$, the $k$ th component of $X$ is denoted by $X_{k}$, and we state $X^{+}=\left\{k, X_{k}=+\right\}, X^{-}=\left\{k, X_{k}=-\right\}$ and $X^{0}=\left\{k, X_{k}=0\right\}$.

We do not give classical definitions, which can be found in the reference book [1]. A motivating example for the matroid notion is given by realizable matroids: any sequence $V=\left(v_{1}, v_{2}, \ldots, v_{k}\right)$ of vectors of $\left(\mathbb{R}^{d}\right)^{*}$ induces the set of covectors $\mathcal{L}(V)=\{(\operatorname{sign}(c$. $\left.\left.\left.v_{1}\right), \operatorname{sign}\left(c \cdot v_{2}\right), \ldots, \operatorname{sign}\left(c \cdot v_{k}\right)\right), c \in \mathbb{R}^{d}\right\}$ (the product used is the classical scalar product in $\left.\mathbb{R}^{d}\right)$.

2.3.1. Oriented Matroid Induced by a Zonotope. For the zonotope $Z(V, M)$, we use the sequence $V_{M}$ of length $s$, obtained by first repeating the vector $v_{1}, m_{1}$ times, then repeating the vector $v_{2}, m_{2}$ times, and so on until repeating the vector $v_{D}, m_{D}$ times. The oriented matroid $\mathcal{L}(Z(V, M))$ is the realizable matroid $\mathcal{L}\left(V_{M}\right)$. It is a matroid of rank $d$.

We have a direct interpretation of $\mathcal{L}(Z(V, M))$ : for each element $X=\left(v_{1}^{\prime}, v_{2}^{\prime}, \ldots, v_{s}^{\prime}\right)$ of $\mathcal{L}(Z(V, M))$ the set $Z_{X}=\sum_{i \in X_{0}}\left[-v_{i}^{\prime},+v_{i}^{\prime}\right]+\sum_{i \in X_{+}} v_{i}^{\prime}-\sum_{i \in X_{-}} v_{i}^{\prime}$ is a face of $Z(V, M)$ and, conversely, for each face $f$ of $Z(V, M)$ there exists a unique $X$ of $\mathcal{L}(Z(V, M))$ such that $Z_{x}=f$.

2.3.2. Oriented Matroid Induced by a Tiling. Given a tiling $T$, a sign vector of length $s$ can be associated with each face of a tile of $T$. For each pair $\left(v_{i}, j\right)$ such that $1 \leq j \leq m_{i}$, we state $k(i, j)=\sum_{1 \leq i^{\prime}<i} m_{i^{\prime}}+j$. Let $f$ be a face and let $X^{f}$ denote the sign vector associated with the face $f$. We have

- $X_{k(i, j)}^{f}=+$ when $f$ is contained in $T_{\left\{v_{i}, j\right\}}^{+}$,

- $X_{k(i, j)}^{f}=-$ when $f$ is contained in $T_{\left\{v_{i}, j\right\}}^{-}$,

- $X_{k(i, j)}^{f}=0$ otherwise.

The set of all these sign vectors is denoted by $\mathcal{O}(T)$.

The set $\mathcal{L}(T)=\{(X,+), X \in \mathcal{O}(T)\} \cup\{(-X,-), X \in \mathcal{O}(T)\} \cup\{(X, 0), X \in$ $\mathcal{L}(Z(V, M))\}$ is the set of covectors of an oriented matroid of rank $d+1$, which will be called the tiling matroid.

In matroid language, a flip is a specific mutation (see Section 7.3 of [1]) of the tiling matroids which only involves cocircuits corresponding with tiles (i.e. cocircuits whose last component is not null).

\section{Decomposition and Reconstruction}

\subsection{Deletions}

3.1.1. Geometrical Deletion. The deletion is a basic operation in matroid theory. We first present it in a geometrical point of view. 
Let $T$ be a tiling of support $Z(V, M)$, and let $S_{\left\{v_{i}, j\right\}}$ be a de Bruijn zone of $T$. One can remove the tiles of $S_{\left\{v_{i}, j\right\}}$ and translate all the tiles of $T_{\left\{v_{i}, j\right\}}^{+}$by the vector $-v_{i}$. For $D>d$, the configuration obtained is a tiling of $Z^{\prime}=\left(V, M^{\prime}\right)$ where $M^{\prime}$ is defined by $m_{i}^{\prime}=m_{i}-1$ and $\forall k \neq i, m_{k}^{\prime}=m_{k}$ (except in the special case when $m_{i}=1$, in such a case we have $Z^{\prime}=\left(V^{\prime}, M^{\prime}\right)$ with $V^{\prime}$ and $M^{\prime}$ respectively obtained from $V$ and $M$ by removing the $i$ th component). Such an operation defines a deletion relation on zonotope tilings.

The tiling obtained is denoted by $D_{\left\{v_{i}, j\right\}}(T)$, and for each tile $t$ of $T$, we state $D_{\left\{v_{i}, j\right\}}(t)=t$ for $t$ in $T_{\left\{v_{i}, j\right\}}^{+}$, and $D_{\left\{v_{i}, j\right\}}(t)=t-v_{i}$ for $t$ in $T_{\left\{v_{i}, j\right\}}^{+}$.

For consistency, the de Bruijn zones of $D_{\left\{v_{i}, j\right\}}(T)$ according to $v_{i}$ are assumed to be numbered $1,2, \ldots, j-1, j+1, \ldots, m_{i}$. In this way, $D_{\left\{v_{i}, j\right\}}(t)$ and $t$ are both in de Bruijn zones with the same label. We also need this convention for the commutativity below, when $v_{i}=v_{k}$.

3.1.2. Deletions in Matroid Theory. As has been said above, deletions are classical in matroid theory. If $\mathcal{L}$ is a set of covectors of length $n$, then for each integer $i$ such that $1 \leq i \leq n$, the set $\mathcal{L} / i=\left\{\left(X_{1}, X_{2}, \ldots, X_{i-1}, X_{i+1}, \ldots, X_{n}\right), X \in \mathcal{L}, X_{i}=0\right\}$ is also a set of covectors.

Thus, in matroid language, the geometrical deletion above consists in constructing $\mathcal{L}(T) / k(i, j)$ from $\mathcal{L}(T)$. Notice that $\mathcal{L}(T) /(s+1)=\mathcal{L}(Z(V, M))$. The Bohne-Dress theorem [14] claims that the only sets $\mathcal{L}$ of covectors of uniform matroids such that $\mathcal{L} /(s+1)=\mathcal{L}(Z(V, M))$ are tiling matroids.

\subsection{Decomposition}

Proposition 3.1 (Commutativity of Deletions). Let $T$ be a tiling of a zonotope $Z$, and let there be two deletions $D_{\left\{v_{i}, j\right\}}$ and $D_{\left\{v_{k}, l\right\}}$. We have

$$
D_{\left\{v_{i}, j\right\}}\left(D_{\left\{v_{k}, l\right\}}(T)\right)=D_{\left\{v_{k}, l\right\}}\left(D_{\left\{v_{i}, j\right\}}(T)\right) .
$$

See Fig. 3.

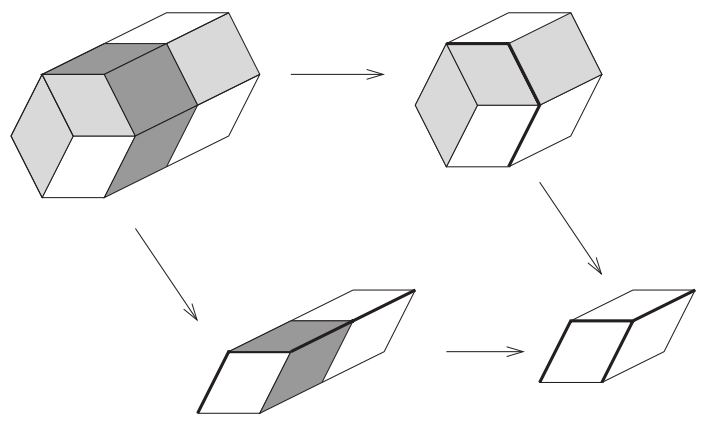

Fig. 3. Commutativity of deletions. 
This proposition is obvious in the matroid framework, but following the principle of the paper, we give a geometrical proof.

Proof. The tiling $T$ can be partitioned into the five parts below:

- $T_{\left\{v_{i}, j\right\}}^{-} \cap T_{\left\{v_{k}, l\right\}}^{-}$: the tiles of this part remain unchanged by the successive deletions, taken in any order.

- $T_{\left\{v_{i}, j\right\}}^{+} \cap T_{\left\{v_{k}, l\right\}}^{-}$: the tiles of this part are translated by $-v_{i}$ during the successive deletions, taken in any order.

- $T_{\left\{v_{i}, j\right\}}^{-} \cap T_{\left\{v_{k}, l\right\}}^{+}$: the tiles of this part are translated by $-v_{k}$ during the successive deletions, taken in any order.

- $T_{\left\{v_{i}, j\right\}}^{+} \cap T_{\left\{v_{k}, l\right\}}^{+}$: the tiles of this part are translated by $-\left(v_{i}+v_{k}\right)$ during the successive deletions, taken in any order.

- $S_{\left\{v_{i}, j\right\}} \cup S_{\left\{v_{k}, l\right\}}$ : the tiles of this part are removed during the successive deletions, taken in any order.

Thus the order of deletions does not give any change. This gives the commutativity result.

A tiling obtained from $T$ by a sequence of $p$ deletions is called an $(s-p)$-minor of $T$.

The pairs $\left\{v_{i}, j\right\}$ can be totally ordered (using the integer $k(i, j)$, for example). From this order, the sets $\left\{\left\{v_{i_{1}}, j_{1}\right\},\left\{v_{i_{2}}, j_{2}\right\}, \ldots,\left\{v_{i_{p}}, j_{p}\right\}\right\}$ formed by $p$ elements of the type $\left\{v_{i}, j\right\}$ can also be totally ordered. Therefore, the $(s-p)$-minors of $T$ can be totally ordered. The sequence of $(s-p)$-minors of $T$ is given by this order.

Proposition 3.2 (Encoding). Assuming $s \geq d+2$, every tiling is defined by the sequence of its (s-1)-minors.

Proof. Let $Z$ be a zonotope, and let $T$ be one of its tilings. Notice that one can easily compute the sequence of $(s-2)$-minors of $T$ from the sequence of its $(s-1)$-minors.

Let $\left\{v_{i}, j\right\}$ and $\left\{v_{i^{\prime}}, j^{\prime}\right\}$ be two distinct pairs, let $D_{1}$ and $D_{2}$ respectively denote the corresponding deletions and let $D_{1,2}$ denote the corresponding double deletion.

For each tile $t^{\prime}$ of $D_{1,2}(T)$, one can easily compute the tiles $t_{1}$, such that $t_{1}$ is in $D_{2}(T)$ and $D_{1}\left(t_{1}\right)=t^{\prime}$, and $t_{2}$, such that $t_{2}$ is in $D_{1}(T)$ and $D_{2}\left(t_{2}\right)=t^{\prime}$. Precisely, one can compute the pair $\left(\varepsilon_{1}, \varepsilon_{2}\right)$ of $\{0,1\}^{2}$ such that $t_{1}=t^{\prime}+\varepsilon_{1} v_{i}$ and $t_{2}=t^{\prime}+\varepsilon_{2} v_{i^{\prime}}$.

Let $t_{0}$ be the tile of $T$ such that $t_{1}=D_{2}\left(t_{0}\right)$. From the commutativity, we also have $t_{2}=D_{1}\left(t_{0}\right)$ (see Fig. 4), in such a way that $t^{\prime}=D_{2}\left(D_{1}\left(t_{0}\right)\right)$. Thus there exists a pair $\left(\varepsilon_{3}, \varepsilon_{4}\right)$ of $\{0,1\}^{2}$ such that $t_{0}=t_{1}+\varepsilon_{4} v_{i^{\prime}}$ and $t_{0}=t_{2}+\varepsilon_{3} v_{i}$. Thus, by composition, we obtain $t_{0}=t^{\prime}+\varepsilon_{1} v_{i}+\varepsilon_{4} v_{i^{\prime}}$ and $t_{0}=t^{\prime}+\varepsilon_{2} v_{i^{\prime}}+\varepsilon_{3} v_{i}$.

These equalities imply that $\varepsilon_{1}=\varepsilon_{3}$ and $\varepsilon_{2}=\varepsilon_{4}$, when $v_{i} \neq v_{i^{\prime}}$. When $v_{i}=v_{i^{\prime}}$, these equalities are obtained by a case by case analysis according to the relative position of $t_{0}$ and the considered de Bruijn zones, as in the proof of Proposition 3.1. Thus we have the equality $t_{0}=t_{2}+\varepsilon_{1} v_{i}=t^{\prime}+\varepsilon_{1} v_{i}+\varepsilon_{2} v_{i^{\prime}}$, which proves that $t_{0}$ can be computed from the triple $\left(t^{\prime}, t_{1}, t_{2}\right)$.

This gives the result, since, for each tile $t$ of $T$, there are two distinct pairs $\left\{v_{i}, j\right\}$ and $\left\{v_{i^{\prime}}, j^{\prime}\right\}$ such that $t$ is out of $S_{\left\{v_{i}, j\right\}} \cup S_{\left\{v_{i}, j^{\prime}\right\}}$ (from the hypothesis $s \geq d+2$ ). 


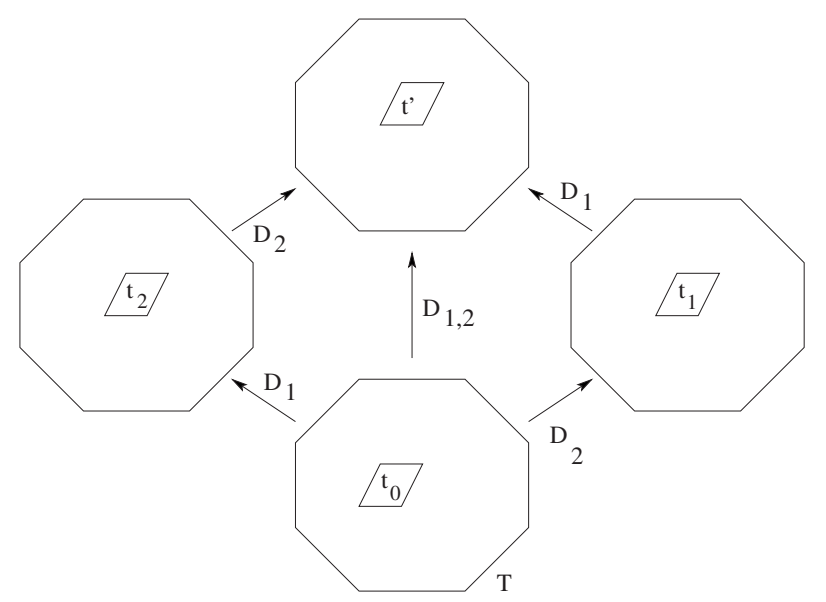

Fig. 4. Proof of Proposition 3.2: computation of some tiles of $T$ from $D_{2}(T)$ and $D_{1}(T)$.

The same result can easily be obtained with chirotopes (Section 3.5 of [1]) using matroid theory (especially the theorem from Lawrence Section 3.5.5 of [1] which characterizes oriented matroids by chirotopes): the knowledge of minors allows us to compute the whole chirotope of the matroid whose set of covectors is $\mathcal{L}(T)$.

Notice that the result is false for $s=d+1$. Each $d$-minor is reduced to a single tile, thus the information about the arrangement of tiles is lost.

Iterating the proof for $(s-1)$-deletion, one obtains the following result as a corollary for Proposition 3.2 (see Fig. 3).

Corollary 3.3. Let $s^{\prime}$ be an integer such that $d+1 \leq s^{\prime} \leq s$. Assuming $s \geq d+2$, every tiling $T$ of a zonotope is defined by the sequence of its $s^{\prime}$-minors. In particular, this is true for $(d+1)$-minors.

Proof. Obvious by induction.

Note that there are two kinds of $(d+1)$-minors: those of codimension 0 , the forced minors, which are defined by the tiled zonotopes, and those of codimension 1, the free minors, which are tilings of unitary zonotopes. Only the free ones contain some information useful to compute $T$. Those encoded by free minor information can be reduced to a single bit, corresponding to the tiling chosen (recall that a $(d+1)$-unitary zonotope admits two tilings).

This gives an encoding of zonotope tilings by a word on the alphabet $\{0,1\}$ of length $\sum_{1 \leq i_{1}<i_{2}<\cdots<i_{d} \leq D} m_{i_{1}} m_{i_{2}} \cdots m_{i_{d}}$ (see Fig. 5 for an example).

We define a set flip as follows: let $T$ and $T^{\prime}$ be two tilings of a same zonotope such that all their $d+1$-minors are the same, except one. We say that $T$ and $T^{\prime} \operatorname{differ}$ by a set flip.

Proposition 3.4. Let $T$ and $T^{\prime}$ be two tilings of a zonotope $Z$. $T$ differs from $T^{\prime}$ by a set flip if and only if $T$ differs from $T^{\prime}$ by a geometric flip. 

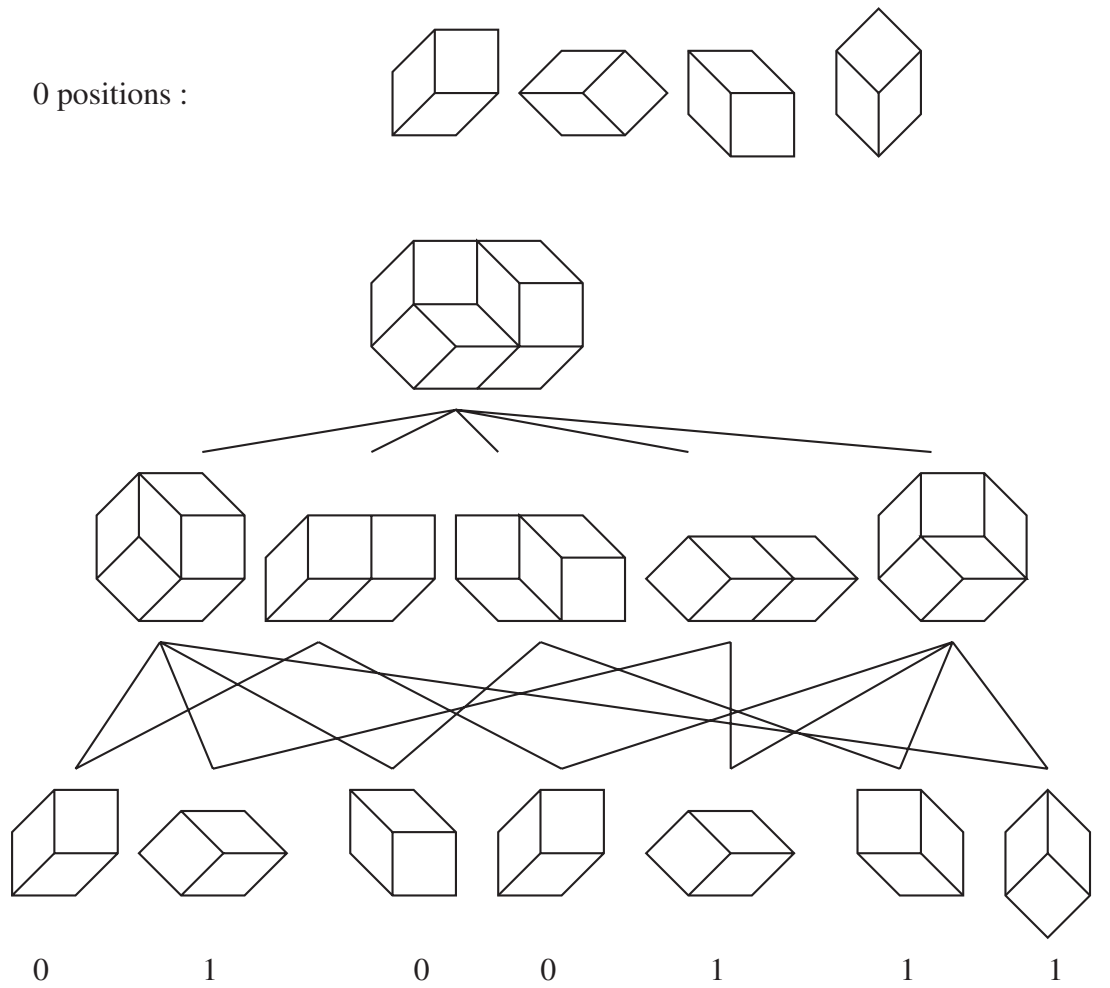

Fig. 5. Coding of tilings with $(d+1)$-minors: each tiling of a $(d+1)$-zonotope is given a bit (tilings on the top corresponding to 0 bits) and the tiling is given by the sequence of bits of its minors.

Proof. It is clear that a geometric flip is a set flip, because it changes locally the positions of $d+1$ tiles. Since only one $(d+1)$-minor contains all these tiles, their positions are changed only in this minor.

For the converse part, we first study how a deletion and a set flip act on a fixed de Bruijn line $d B L$. A deletion (which does not remove the whole de Bruijn line $d B L$ ) only removes one tile of $d B L$ and does not change the order in this line for the other tiles. Thus a set flip changes the order on $d B L$ if and only if $d B L$ contains a pair $\left\{t, t^{\prime}\right\}$ of tiles which appear in the flip. Moreover, in this case, the comparison order in $d B L$ is changed only for the pair $\left\{t, t^{\prime}\right\}$, since any other pair of tiles appears in a $(d+1)$-minor unchanged by the flip. Thus, for consistency of the order, the tiles $t$ and $t^{\prime}$ necessarily share a whole facet. Thus the flip is actually geometric.

This property is a special case of the equivalence between mutations in oriented matroids and flippings in arrangements of pseudospheres [1, Section 7.3] (recall that the combinatorial structure of oriented matroids is equivalent to the topological structure of arrangements of pseudospheres of the topological representation theorem of Folkman and Lawrence [1, Section 1.4.1]). 


\subsection{Reconstruction}

We are interested in the following problem: given a zonotope $Z$ and a sequence of $(d+1)$ tilings (with a good length and good vectors), does there exist a tiling $T$ of $Z$ such that the given sequence is the sequence of its $(d+1)$-minors?

We can obviously solve the problem by constructing the (potential) $(d+2)$-minors, then the $(d+3)$-minors and so on until the required tiling is found. If there is a contradiction, the reconstruction is impossible, otherwise the tiling is obtained. However, this can give the answer faster, and the following proposition states that the first step is enough to obtain the answer.

Proposition 3.5. Let $(\mathrm{Zm})_{m}$ be a sequence of $(d+1)$-tilings. There exists a tiling $T$ of a zonotope $Z$ whose sequence of $(d+1)$-minors is exactly $(\mathrm{Zm})_{m}$ if and only if the $(d+2)$ minors are compatible, i.e. the sequence of $(d+2)$-minors can be correctly constructed.

Proof. We do the proof by induction on the size $s$ of the zonotope. The case $s=d+2$ is obvious.

Let $s>d+2$. Consider the prefix of the sequence $(\mathrm{Zm})_{m}$ formed by $(d+1)$-tilings where the tiles of the (potential) de Bruijn zone $\left\{v_{D}, m_{D}\right\}$ do not appear (since it is assumed that the deletion $D_{\left\{v_{D}, m_{D}\right\}}$ has been done). This subsequence is, by assumption, the sequence of $(d+1)$-minors of a tiling $T^{\prime}$ of size $s-1$.

On the other hand, for each tile $t$ of $T^{\prime}$, there exists a $(d+1)$-tiling $T_{t}$ containing $t$ and $d$ tiles of the de Bruijn zone $\left\{v_{D}, m_{D}\right\}$. Hence $t$ can be assigned a + or - sign, depending on its position in $T_{t}$, relative to $S_{\left\{v_{D}, m_{D}\right\}}$ ( + if the $D$-located height function of $t$ is 1 , if it is 0 ). Let $T^{\prime+}$ be the part of $T^{\prime}$ formed by the tiles marked + and let $T^{\prime-}$ be the part formed by tiles marked - .

We now consider a straight line $l$ directed by $v_{D}$. We claim that, following $l$ in the sense of $v_{D}$, one first meets tiles marked - , then tiles marked + . This means that $T^{\prime+}$ and $T^{\prime-}$ are convex along $v_{D}$, i.e. that the new de Bruijn zone can be inserted correctly in $T^{\prime}$, thus leading to a new tiling $T$. Two cases may occur:

- $l$ only meets facets and interior part tiles of $T^{\prime}$. Consider two tiles of $T^{\prime}$, say $t_{1}$ and $t_{2}$, which share a facet, and such that $t_{2}$ follows $t_{1}$ in the succession of tiles crossed by $l$ in the direction of $v_{D}$. There exists a $(d+2)$-minor $T_{d+2}$ containing (tiles corresponding to) tiles of $\left\{v_{D}, m_{D}\right\}$ and tiles $t_{1}$ and $t_{2}$. There are only three possible sign assignments for $\left(t_{1}, t_{2}\right)$, since the assignment + for $t_{1}$ and - for $t_{2}$ is impossible; otherwise the tile $t_{3}$ of type $\{\tau\} \cup\left\{v_{D}\right\}$ (where $\tau$ denotes the set of common vectors in the types of $t_{1}$ and $t_{2}$ ) cannot be placed in the $(d+2)$-minor $T_{d+2}$ (see Fig. 6).

- $l$ meets a face $f$ of the tiling $T^{\prime}$ of a dimension lower than $d-1$. Then there are two tiles $t_{1}$ and $t_{2}$ with the same hypothesis as in the previous case, but sharing only the face $f$. There exists a parallel line $l^{\prime}$, arbitrarily close to $l$, satisfying the hypothesis of the previous case, and crossing both $t_{1}$ and $t_{2}$ (but $t_{1}$ and $t_{2}$ are not necessarily consecutive along $l^{\prime}$ ). (See Fig. 7.) Thus the assignment + for $t_{1}$ and for $t_{2}$ is impossible. 

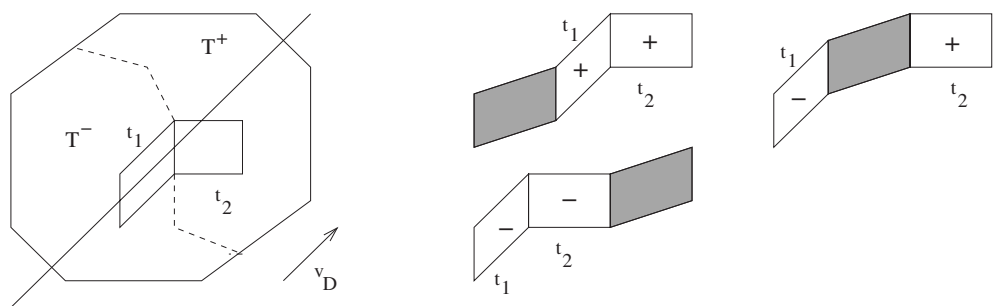

Fig. 6. The three possible sign assignments for $t_{1}$ and $t_{2}$, from the possible positions in the de Bruijn line of $T_{d+2}$.

Hence $T^{\prime+}$ and $T^{\prime-}$ are consistent according to $\left\{v_{D}, m_{D}\right\}$, allowing us to translate the part $T^{\prime+}$ by $v_{D}$, in order to insert the de Bruijn zone $S_{\left\{v_{D}, m_{D}\right\}}$. The tiling $T$ obtained (such that $T^{\prime+}=T_{\left\{v_{D}, m_{D}\right\}}^{+}+v_{D}$ and $T^{\prime-}=T_{\left\{v_{D}, m_{D}\right\}}^{-}$) is the one sought, which ends the proof.

The proposition above is closely linked with the main axiom of the definition of chirotopes [1, Section 3.5.3], which involves sets of $d+2$ indexes. An alternative strategy for proving the proposition is to check that the chirotope axioms are satisfied, or, in a more direct way, to check the local realizability property [1, Section 3.6.3].

\section{Orders and Representation}

As seen previously, the zonotopal tilings can be easily encoded by considering their minors. More precisely, one tiling is defined by the sequence of its (free) $(d+1)$-minors. We now describe a representation tool for zonotope tilings based on the minor structure and the reconstruction proposition. However, before doing it, we have to defines the orders induced by flips, and we need some more knowledge about the space of tilings of $(d+2)$-zonotopes.

\subsection{Orders on Tilings}

If we have $D$ vectors, we arbitrarily fix a basic tiling $T_{0}$ of the unitary $D$-zonotope. For each $(d+1)$-unitary zonotope, we define the low position as the $(d+1)$-tiling of this zonotope which is a $(d+1)$-minor of $T_{0}$. The other $(d+1)$-tiling is the high position.

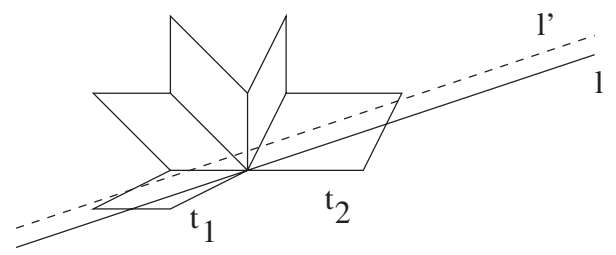

Fig. 7. A line $l$ crossing a vertex, and the auxiliary line $l^{\prime}$. 
Usually, the tiling $T_{0}$ chosen is constructed inductively as follows: given a sequence $\left(v_{1}, v_{2}, \ldots, v_{D}\right)$ of vectors, we define, for each integer $i$ such that $d \leq i \leq D$, the tiling $T_{i}$ of the unitary zonotope $Z_{i}$ constructed on the $i$ first vectors by

- the tiling $T_{d}$ is the unique tiling of $Z_{d}$,

- for $d \leq i<D, T_{i+1}$ is the unique tiling of $Z_{i+1}$ such that $T_{\left\{v_{i+1}, 1\right\}}^{+}$is empty and $D_{\left\{v_{i+1}, 1\right\}}\left(T_{i+1}\right)=T_{i}$.

With this definition, flips can be canonically directed: a flip is going upwards if it transforms a low position into a high position. The directed space of tilings is the space of tilings whose edges are directed as above. Obviously, it is acyclic and defines a partial order relation, denoted by $<_{\text {flip }}$. Given a pair $\left(T, T^{\prime}\right)$ of tilings, we have $T \leq_{\text {flip }} T^{\prime}$ if one can pass from $T$ to $T^{\prime}$ by a sequence of upwards flips.

We can also define another order relation, denoted by $<_{\text {set. }}$. For each tiling $T$, we denote the set of its low $(d+1)$-minors by low $(T)$. Given a pair $\left(T, T^{\prime}\right)$ of tilings, we have $T \leq_{\text {set }} T^{\prime}$ if low $(T) \leq_{\text {set }} \operatorname{low}\left(T^{\prime}\right)$. Obviously, $T \leq_{\text {flip }} T^{\prime}$ yields $T \leq_{\text {set }} T^{\prime}$.

We recall that tilings are single elements liftings of the zonotope matroid. By duality, there is a one-to-one correspondence between these liftings, and single element extensions of the dual matroid [1, Section 3.4]. These extensions are studied in Section 7.1 of [1] and can be canonically ordered. Here, we introduce this order with a geometrical point of view.

The higher Bruhat orders are special cases of these orders for unitary zonotopes with a specific sequence $V$ of vectors, such that, for each subsequence $\left(v_{i_{1}}, v_{i_{2}}, \ldots, v_{i_{d}}\right)$, the determinant $\operatorname{det}\left(v_{i_{1}}, v_{i_{2}}, \ldots, v_{i_{d}}\right)$ is positive.

\subsection{The Basic $(d+2)$-Zonotopes}

In dimension $d$ there exist two basic kinds of $(d+2)$-zonotopes of dimension $d$ whose tiling is not forced: either all vectors have multiplicity 1 (codimension 2), or there is one vector of multiplicity 2 (codimension 1 ). We first study these cases precisely. This should have been done easily in the matroid framework by studying single element extensions of very basic matroids.

\subsubsection{The $(d+2)$-Zonotope of Codimension 1}

Proposition 4.1. The space of tilings of the zonotope $Z_{i}$ of codimension 1 with the vector $v_{i}$ of multiplicity 2 (and the $d$ other ones of multiplicity 1) contains three tilings and is a chain of length 2 .

Proof. In each tiling, there exists a unique tile $t$ which is not an element of $S_{\left\{v_{i}, 1\right\}} \cup S_{\left\{v_{i}, 2\right\}}$. Since $T_{\left\{v_{i}, 1\right\}}^{-} \subseteq T_{\left\{v_{i}, 2\right\}}^{-}$, we have three tilings:

- one tiling with $t \in T_{\left\{v_{i}, 1\right\}}^{-}$,

- one tiling with $t \in T_{\left\{v_{i}, 2\right\}}^{+}$,

- one tiling with $t \in T_{\left\{v_{i}, 2\right\}}^{-} \backslash T_{\left\{v_{i}, 1\right\}}^{-}$. 


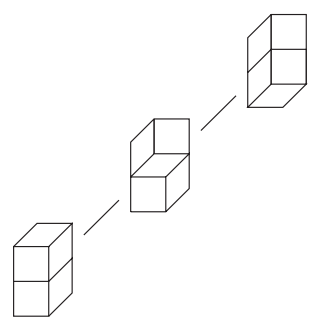

Fig. 8. Space of tilings of a codimension 1 zonotope with one duplicated vector.

The directed edges corresponding to flips are obvious (note that both the free $(d+1)$ minors of $Z_{i}$ are of the same type, which gives the chain) (see Fig. 8).

4.2.2. The Unitary $(d+2)$-Zonotope. We first need more information about the structure of tilings of unitary $(d+1)$-zonotopes. This is given by the lemma below.

Lemma 4.2. Let $T$ be a tiling of a unitary $(d+1)$-zonotope, and let $v$ be a vector not in the type of $Z$. We define a tournament $G_{(T, v)}$ on the tiles of $T$ saying that $\left(t_{1}, t_{2}\right)$ is an arc of $G_{(T, v)}$ if the vector $v$ crosses their common facet passing from $t_{1}$ to $t_{2}$ (see Fig. 9). The tournament $G_{(T, v)}$ is actually a total order.

Proof. Since all pairs of tiles are linked, we only have to prove that $G_{(T, v)}$ has no cycle of length 3 . We prove it by reducing the problem to the case $d=2$, for which the proof is easy by a case-by-case analysis.

In higher dimensions, notice that, since $D=d+1$, the types of three given tiles $t_{1}$, $t_{2}$ and $t_{3}$ contain $(d+1)-3=d-2$ common vectors. Let $p$ denote the orthogonal projection on the two-dimensional space which is orthogonal to the $d-2$ common vectors. The projections $p\left(t_{i}\right)$ are parallelograms, and we have $\left(t_{i}, t_{j}\right)$ in $G_{(T, v)}$ if and only if $\left(p\left(t_{i}\right), p\left(t_{j}\right)\right)$ is in $G_{H, p(v)}$; where $H$ denotes the hexagon covered by the parallelograms $p\left(t_{i}\right)$ ( $H$ is really a hexagon, since otherwise the tiles $t_{i}$ cannot be pairwise adjacent). This gives the result, since $G_{p(v)}$ is not a cycle.

Proposition 4.3. The space of tilings of a unitary $(d+2)$-zonotope is a cycle of length $2(d+2)$, and each possible label is given to a pair of edges, which are opposite in the cycle (see Fig. 10).
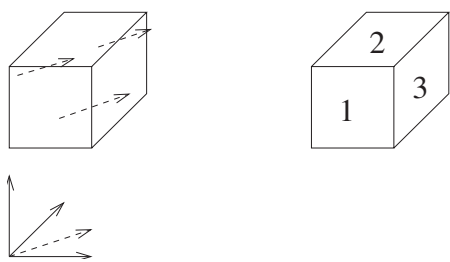

Fig. 9. A codimension 1 tiling, the added vector (dashed), and the ordering of tiles according to this vector. 


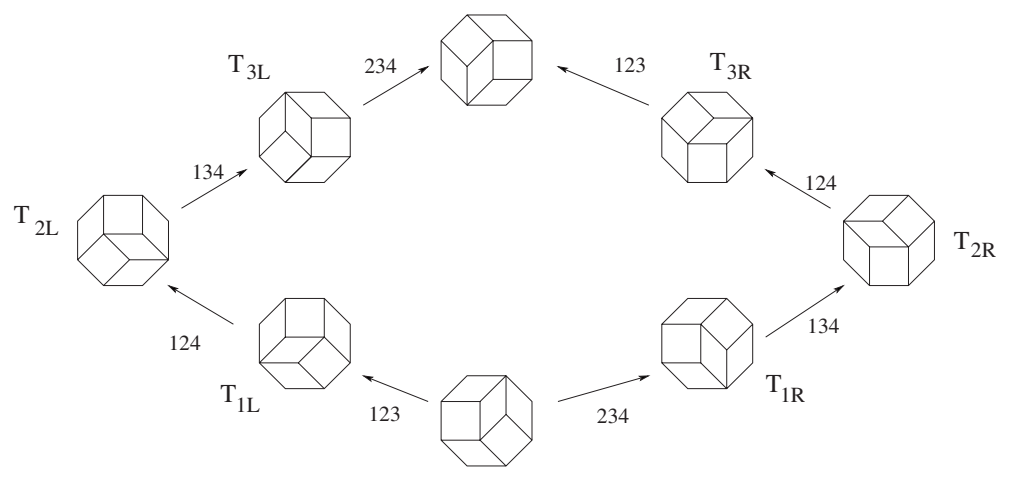

Fig. 10. The order associated with a unitary octagon.

Proof. Let $T$ be a tiling of the unitary zonotope $Z=Z\left(\left(v_{1}, v_{2}, \ldots, v_{d+2}\right),(1,1, \ldots, 1)\right)$. From the above lemma applied on the support $Z^{\prime}$ of $D_{\left\{v_{d+2}, 1\right\}}(T), T_{\left\{v_{d+2}, 1\right\}}^{-}$is an initial segment of the order induced by $v_{d+2}$ on tiles of $D_{\left\{v_{d+2}, 1\right\}}(T)$.

Conversely, given a tiling $T^{\prime}$ whose support is $Z^{\prime}$, and an initial segment $T^{\prime}$ (according to the order induced by $v_{d+2}$ ), one easily constructs a tiling of $Z$ : tiles which are not in the initial segment are translated by $v_{d+2}$, and tiles of $S_{\left\{v_{d+2}, 1\right\}}$ are inserted in the remaining space. There exist $d+2$ possible initial segments for a set of $d+1$ elements, thus, since $Z^{\prime}$ admits two tilings, there exist $2(d+2)$ tilings of $Z$.

Now, take a tiling of $T$, i.e. a tiling $T^{\prime}$ of the zonotope $Z^{\prime}$ and $I$, one initial segment of it. What are the possible flips from $T$ ? First assume that the initial segment is proper (i.e. neither empty nor equal to $T^{\prime}$ ). There are two possible flips, which correspond to adding or removing one tile in $I$. No other flip is possible because of the relative position of tiles given by the order on tiles of $T^{\prime}$ (the flip only using tiles of $T^{\prime}$ is not possible because of the cut by $S_{\left\{v_{d+2}, 1\right\}}$ ).

A similar argument holds for the other case. If $I$ is empty, two flips are possible, one which corresponds to adding the first tile in $I$, and the other which only uses tiles of $T^{\prime}$. If $I=T^{\prime}$, two flips are possible, one which corresponds to removing the last tile in $I$, and the other which only uses tiles of $T^{\prime}$. This gives the result, using the symmetry of both tilings of $Z^{\prime}$ to get the labels of opposite edges.

\subsection{Tiling Diagrams}

We can now explain precisely how we represent a fixed tiling $T$. There exist some related representations in [19] about matroids, which are consequences of the topological representation theorem for matroids [1, Section 1.4.1] and of the use of signatures of single element extensions [1, Section 7.1]. A specific originality of our representation consists in the use of arrows to treat parallelism.

4.3.1. Points. In our representation, each $(d+1)$-minor is associated to a point. Each point $p$ is defined by two parameters. We first have a coordinate vector, an element of 
$\mathbb{Z}^{D}$, which indicates the position of the $(d+1)$-minor in the sequence of minors: the $i$ th component, denoted by $i(p)$, of this vector is equal to $j$ if the deletion according to the pair $\left\{v_{i}, j\right\}$ has not been done to obtain the corresponding $(d+1)$-minor; the component $i(p)$ is null if, for each integer $j$ such that $1 \leq j \leq m_{i}$, the deletions according to $\left\{v_{i}, j\right\}$ have been done (thus there exists exactly $d+1$ non-null components). The type of the corresponding flip is the support of the coordinate vector.

Note that a similar coordinate vector will also be given to each $d^{\prime}$-minor whose support is a unitary zonotope: the only difference is that there are $d^{\prime}$ non-null components.

For each such unitary zonotope $Z_{d^{\prime}}$, we define the space of points associated with $Z_{d^{\prime}}$ as the set of points which corresponds to minors of $Z_{d^{\prime}}$. We say that $d^{\prime}-d-1$ is the dimension of this space. In particular, for $d^{\prime}=d+2$, we speak of the line associated with $Z_{d+2}$. In order to justify our vocabulary, we can see that each line is defined by two points (but, unfortunately, each pair of points does not always define a line).

The other parameter is a color, which is white if the $(d+1)$-minor is in a low position, or black if in a high position.

The important thing for reconstructing a tiling $T$ is the set of coloring constraints which are given by the sequence of $(d+2)$-minors. We now explain how coloring constraints are expressed.

4.3.2. Arrows. Two points correspond to the pair of minors of a same $(d+2)$-minor of $T$ (the support of this $(d+2)$-minor is a $(d+2)$-zonotope of codimension 1) if and only if they only differ by one non-null coordinate.

From what has been seen about these $(d+2)$-tilings, there exist exactly three allowed colorings of such a pair of points, corresponding to tilings of a $(d+2)$-zonotope, and a forbidden coloring corresponding with no tiling.

The forbidden coloring uses both colors. In the diagram (see Fig. 11 for an example), an arrow linking these two points is placed in such a way that the origin of the arrow is white (and the tail is black) in the forbidden coloring. Thus, the three allowed colorings of the tiled $(d+2)$-zonotope are the fully black one, the fully white one and the coloring with the origin of the arrow being black and the tail being white. This gives the first constraint: there is no edge from a white point to a black point.

Each arrow is labeled by the index of the coordinate which is different for the points linked.

The arrows of the diagram give the covering relation: a point $p$ is covered by a point $p^{\prime}$ if there exists an arrow such that $p$ is the origin of the arrow and $p^{\prime}$ is its endpoint.

4.3.3. Lines. Now consider a $(d+2)$-minor of $T$ whose support is a unitary $(d+2)$ zonotope. A point corresponds to a $(d+1)$-minor of this $(d+2)$-tiling if and only if its coordinate vector is obtained by replacing one non-null coordinate of the $(d+2)$-minor by 0 .

Such points form a line which can be totally ordered in the same way as flips are ordered in a path between the lowest tiling of the unitary $(d+2)$-zonotope to its largest tiling. (There are two possible opposite sequences, but this fact is not relevant, one of these can be chosen arbitrarily. Later we will choose a specific order in a special case.) From what has been seen about tilings of unitary $(d+2)$-zonotopes, with the order convention, the black points have to form a final or initial segment (i.e. a suffix or a prefix) of the line. This is the second constraint. 


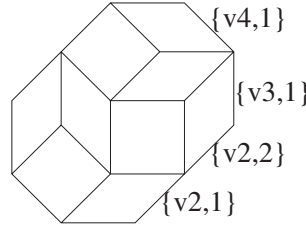

$\{\mathrm{v} 1,1\}$

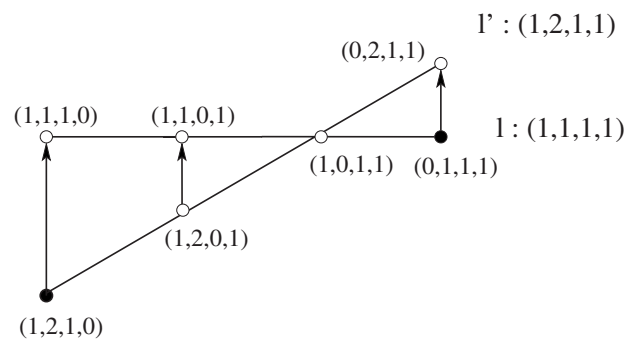

Fig. 11. A tiling and the associated diagram (notice the orientation of the arrows, according to the inversion property).

Hence, tilings of zonotopes are presented as diagrams on which lines represent unitary $(d+2)$-zonotopes, and arrows represent $(d+2)$-zonotopes of codimension 1 (see Fig. 11). Notice that arrows and lines only depend on the support of the tiling, i.e. two tilings with the same support induce the same sets of lines and arrows. Translation of the reconstruction theorem gives the following result:

Proposition 4.4. A coloring of points of a diagram induces a tiling if and only if it respects the two constraints stated above.

Notice that the highest diagram (i.e. with all points black) and the lowest one (i.e. with all points white) are tilings. We denote the set of black points of the diagram of $T$ by $B_{T}$. Hence, for each pair $\left(T, T^{\prime}\right)$ of tilings, we have $T \leq_{\text {set }} T^{\prime}$ if and only if $B_{T} \subseteq B_{T^{\prime}}$.

\subsection{Recursivity Properties of Diagrams}

Diagrams have some interesting recursivity properties, which are very useful for induction arguments.

4.4.1. Deletions. Let $T$ be a tiling of a zonotope $Z$, let $\left(v_{i}, j\right)$ be a pair defining a de Bruijn section of $T$ and let $T^{\prime}$ be the tiling such that $T^{\prime}=D_{\left\{v_{i}, j\right\}}(T)$. The diagram of $T^{\prime}$ is obtained from the diagram of $T$ by removing all the points whose $i$ th coordinate is equal to $j$. The remaining line and arrow relations are preserved (we take the convention that the tiling $T_{0}^{\prime}=D_{\left\{v_{i}, 1\right\}}\left(T_{O}\right)$ is used to define the sense of flips).

4.4.2. Contractions. Let $T$ be a tiling of a zonotope $Z$ and let $\left(v_{i}, j\right)$ be a pair defining a de Bruijn section of $T$. Let $q_{i}$ be the orthogonal projection on the hyperplane $\left\{v_{i}\right\}^{\perp}$.

For $d>3$, we define the contracted tiling $T^{\prime}=C_{\left\{v_{i}, j\right\}}(T)$ as follows: $T^{\prime}$ is the tiling formed by the set $\left\{q_{i}(t), t \in S_{\left\{v_{i}, j\right\}}\right\}$. The support of this tiling is the zonotope $Z^{\prime}=\left(V^{\prime}, M^{\prime}\right)$ with $V^{\prime}=\left(q_{i}\left(v_{1}\right), q_{i}\left(v_{2}\right), \ldots, q_{i}\left(v_{i-1}\right), q_{i}\left(v_{i+1}\right), q_{i}\left(v_{i+2}\right), \ldots, q_{i}\left(v_{D}\right)\right)$ and $M^{\prime}=\left(m_{1}, m_{2}, \ldots, m_{i-1}, m_{i+1}, m_{i+2}, \ldots, m_{D}\right)$. Hence, $T^{\prime}$ is a tiling of dimension $d-1$, codimension $D-d$ and size $s-m_{i}$. The contraction geometrically defined above is also a classical operation on matroids. 
How can we find the diagram of $T^{\prime}$ from the diagram of $T$ ? We have to keep only all points whose $i$ th coordinate is $j$. Each line either disappears (if its $i$ th coordinate different from $j$ ) or loses a point. Arrow relations are preserved between all the points which are kept (for orientation convention, the lowest tiling which defines the low positions is $\left.T_{0}^{\prime \prime}=C_{\left\{v_{i}, 1\right\}}\left(T_{0}\right)\right)$.

\subsection{Consistence Properties of Arrows}

We now give two propositions about the relative positions of arrows.

\subsubsection{Same Type Property}

Proposition 4.5. Let $p, p^{\prime}, p_{1}$ and $p_{1}^{\prime}$ be four points of a diagram, such that:

- there exists an integer $i$ such that $0<i(p)<i\left(p^{\prime}\right)$ and $0<i\left(p_{1}\right)<i\left(p_{1}^{\prime}\right)$,

- for each integer $j$ of $\{1,2, \ldots, D\}$ such that $j \neq i$, we have $j(p)=j\left(p^{\prime}\right)$ and $j\left(p_{1}\right)=j\left(p_{1}^{\prime}\right)$. Moreover, if $j(p)=0$, we have $j\left(p_{1}\right)=0$.

There exists an arrow from $p$ to $p^{\prime}$ if and only if there exists an arrow from $p_{1}$ to $p_{1}^{\prime}$.

Proof. This is obvious: the support of the tiling whose minors are $p$ and $p^{\prime}$ is a $(d+2)$ zonotope with mulitiplicity 2 for $v_{i}$, and it is also the tiling whose minors are $p_{1}$ and $p_{1}^{\prime}$.

The inequalities $i(p)<i\left(p^{\prime}\right)$ and $i\left(p_{1}\right)<i\left(p_{1}^{\prime}\right)$ indicate that $p$ and $p_{1}$ both give tilings of minors constructed by the same deletion, as $p^{\prime}$ and $p_{1}^{\prime}$ also do.

\subsubsection{Inversion Property}

Proposition 4.6 (Inversion Property). Let $l=\left(p_{1}, p_{2}, \ldots, p_{d+2}\right)$ and $l^{\prime}=$ $\left(p_{1}^{\prime}, p_{2}^{\prime}, \ldots, p_{d+2}^{\prime}\right)$ be two distinct lines such that there exists a unique integer $k$ such that $p_{k}=p_{k}^{\prime}$. Assume $p_{1}$ is covered by $p_{1}^{\prime}$. For any integer $j$ such that $1 \leq j<k, p_{j}$ is covered by $p_{j}^{\prime}$, and for any integer $j$ such that $k<j \leq d+2, p_{j}^{\prime}$ is covered by $p_{j}$ (see Fig. 11 for an illustration of this property).

Of course, a similar property holds when it is assumed that $p_{d+2}$ is covered by $p_{d+2}^{\prime}$.

Proof. With the notations above, the coordinates vectors of $l$ and $l^{\prime}$ have the same positions for non-null coordinates, that we denote by $i_{1}, i_{2}, \ldots$ and $i_{d+2}$. Moreover, all these non-null coordinates are equal except for one position. We denote this unique position as $i_{0}$.

Consider the $(d+2)$-minor whose sequence of minors corresponds to points $p_{j}$ and $p_{j}^{\prime}$. Its support $Z_{0}$ has codimension 2, its multiplicity is 2 according to the vector $v_{i_{0}}$, and its multiplicity is 1 according to any vector other vector appearing in the type of at least a point.

Consider the tiling $T_{\mathrm{wh}}$ of $Z_{0}$ corresponding to the fully white coloring. This tiling has two minors of codimension 2 which are obtained by a deletion according to $v_{i}$. By definition, both these minors are equal to $T_{0}$. That means that there is no tile between both de Bruijn sections according to $v_{i_{0}}$ of $T_{\mathrm{wh}}$. 


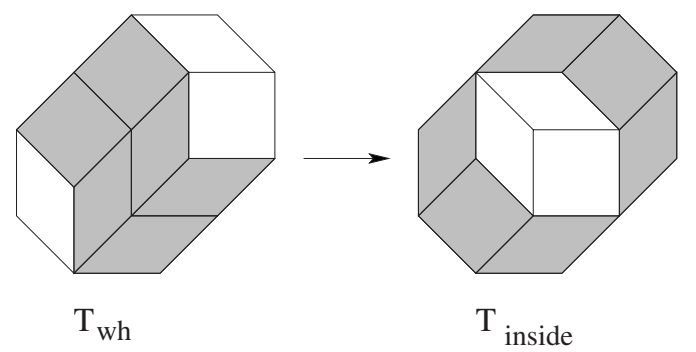

Fig. 12. The tilings $T_{\mathrm{wh}}$ and $T_{\text {inside. }}$.

By a sequence of flips, one can move the de Bruijn sections in such a way that each tile (whose type does not contain $v_{i}$ ) of the resulting tiling $T_{\text {inside }}$ is between both de Bruijn sections according to $v_{i_{0}}$ (see Fig. 12).

The set of black points of $T_{\text {inside }}$ is necessarily one of the sets $\left\{p_{1}, p_{2}, \ldots, p_{i-1}, p_{i+1}^{\prime}\right.$, $\left.p_{i+2}^{\prime}, \ldots, p_{d+2}^{\prime}\right\}$ or $\left\{p_{1}^{\prime}, p_{2}^{\prime}, \ldots, p_{i-1}^{\prime}, p_{i+1}, p_{i+2}, \ldots, p_{d+2}\right\}$. However, the second set is not possible, from our assumption about the arrow from $p_{1}$ to $p_{1}^{\prime}$ (if $p_{1}^{\prime}$ is black, then $p_{1}$ is necessarily black).

Thus the set of black points is $\left\{p_{1}, p_{2}, \ldots, p_{i-1}, p_{d+2}^{\prime}, p_{d+1}^{\prime}, \ldots, p_{i+1}^{\prime}\right\}$, which forces the sense of arrows, and gives the result.

\section{Order Equality in Codimension 2}

In this section we limit ourselves to the case when $D=d+2$. A type of a flip will be labeled by the sequence of indexes of the $d+1$ vectors involved in it, or, for convenience, by the overlined index by the missing vector (for example, a flip whose support is the unitary zonotope constructed with the first $d+1$ vectors is labeled by $12 \cdots d+1=$ $\overline{d+2)}$.

Consider the unitary zonotope induced by the sequence $\left(v_{1}, v_{2}, \ldots, v_{d+2}\right)$. We recall that the space of tilings of this zonotope is formed by two directed chains from the minimal tiling $T_{0}$ to the maximal tiling $T_{1}$, which only meet at their endpoints. Up to vector renumbering, it can be assumed that, from $T_{0}$ to $T_{1}$ the sequence of successive labels in a chain is $(\overline{d+2}, \overline{d+1}, \ldots, \overline{1})$ and the sequence is $(\overline{1}, \overline{2}, \ldots, \overline{d+2})$ for the other chain (see Fig. 10).

We can encode each tiling by its position on this space, as follows: the tiling at the end of the path issued from $T_{0}$, whose sequence of labels is $(\overline{d+2}, \overline{d+1}, \ldots, \overline{d+3-i})$, is denoted by $T_{i \mathrm{~L}}$ (informally, $T_{i \mathrm{~L}}$ is in the $i$ th position on the left chain), and the tiling at the end of the path, issued from $T_{0}$, whose sequence of labels is $(\overline{1}, \overline{2}, \ldots, \bar{i})$, is denoted by $T_{i \mathrm{R}}$ (informally, $T_{i \mathrm{R}}$ is in the $i$ th position on the right chain).

We now use our representation to obtain some structural results on the space of tilings. Our main theorem is stated below:

Theorem 5.1. Let $\left(T, T^{\prime}\right)$ be a pair of tilings with the same support. We have $T \leq_{\text {flip }} T^{\prime}$ if and only if $T \leq \mathrm{set} T^{\prime}$. 
The direct part of the theorem is obvious. To prove the converse, we consider two tilings $T$ and $T^{\prime}$ such that $B_{T} \subseteq B_{T^{\prime}}$ (remember that $B_{T}$ (respectively $B_{T^{\prime}}$ ) is the set of black points of the diagram of $T$ (respectively $T^{\prime}$ )). For convenience, we first introduce some vocabulary. The points in $B_{T}$ are said to be wholly black, those which are not elements of $B_{T^{\prime}}^{\prime}$ are wholly white and the remaining points are positive. A positive point $p$ is removable from the pair $\left(T, T^{\prime}\right)$ if $B_{T^{\prime}} \backslash\{p\}$ is the set of black points of a diagram of a tiling.

We have to prove that (when $T \neq T^{\prime}$ ) there exists a removable point. We decompose this proof into two preliminary steps, each of them corresponding to a type of constraint: the cluster reduction (for arrow constraints) and study of the obstacle graph (for line constraints).

\subsection{Cluster Reduction}

The cluster $F_{p}$ generated by a point $p$ is the set of lines passing through $p$. From Proposition 4.5 , the covering relation induces a total order over the points of $F_{p}$ having the same type. Moreover, since the codimension is 2 , a cluster can be seen as the diagram of a zonotope $Z_{p}$ (with all multiplicities, except one, being unitary), and the previous colorings, restricted to $F_{p}$, are tilings, say $T_{p}$ and $T_{p}^{\prime}$, of $Z_{p}$. Since $B_{T} \subseteq B_{T^{\prime}}$, we have $B_{T_{p}} \subseteq B_{T_{p}^{\prime}}$.

We say that a positive point $p$ is critical if $p$ is removable in $F_{p}$ for the pair $\left(T_{p}, T_{p}^{\prime}\right)$.

A point $p$ is covered by no other point of $F_{p}$. Thus, if a critical point $p$ is covered by no other positive point, then $p$ is removable.

Lemma 5.2. Let $p$ be a critical point and let $p^{\prime}$ be a positive point which covers $p$. The point $p^{\prime}$ is also critical.

Proof. Consider a line $l^{\prime}$ passing through $p^{\prime}$. There exists a line $l$ passing through $p$ and meeting $l^{\prime}$ ( since $p^{\prime}$ covers $p$ they differ by only one coordinate) on a common point $p^{\prime \prime}$. Let $\left(T_{l}, T_{l}^{\prime}\right)$ denote the pair of tilings of the unitary $(d+2)$-zonotope induced by $\left(T, T^{\prime}\right)$ from the colorings of the line $l$. With the inversion property, one sees that the color of $p^{\prime \prime}$ allows us to deduce the color of each point of $l \cup l^{\prime}$.

By an easy case by case analysis on the color of $p^{\prime \prime}$, one deduces that, for each pair $\left(p_{1}, p_{1}^{\prime}\right)$ of $l \times l^{\prime}$ such that $p_{1}$ and $p_{1}^{\prime}$ are linked by an arrow, $p_{1}$ and $p_{1}^{\prime}$ have the same color. Thus if $p$ is removable on $l$ for the pair $\left(T_{l}, T_{l}^{\prime}\right)$, then $p^{\prime}$ is removable on $l^{\prime}$ for the pair $\left(T_{l^{\prime}}, T_{l^{\prime}}^{\prime}\right)$ (see Fig. 13 for an example; one can check that other configurations lead to the same result). Thus $p^{\prime}$ is critical.

Corollary 5.3. If there exists a critical point, then there exists a removable point.

Proof. Let $p_{0}$ be a critical point. If $p_{0}$ is covered by no positive point, then $p_{0}$ is removable. Otherwise, $p_{0}$ is covered by a critical point $p_{1}$, from the previous lemma.

Iterating this process, one can construct a (finite or infinite) path $\left(p_{j}\right) j$ of points such that, for each integer $j, p_{j+1}$ covers $p_{j}$. From Proposition 4.5, such a path cannot pass 


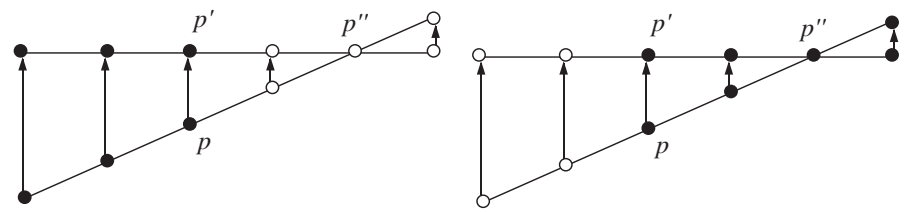

Fig. 13. The two possible colorings in the proof of Lemma 5.2.

by a same point twice, which ensures that the sequence is finite, i.e. the process cannot be repeated infinitely. Thus, there exists a critical point which is covered by no positive point, i.e. a removable point.

\subsection{Obstacle Graph}

We now present the main tool used for the proof of Theorem 5.1.

Definition 3 (Obstacle Graph). The obstacle graph is the labeled directed graph $G$ where:

- The vertices of $G$ are the tilings (or corresponding lines of colored points) of the unitary $(d+2)$-zonotope (except $\left.T_{0}\right)$.

- $\left(T, T^{\prime}\right)$ is an edge of $G$ if there exists a $(d+3)$-tiling $T_{\text {aux }}$ of codimension 2 such that:

- $T$ is a minor of $T_{\text {aux }}$ corresponding to a line $l=\left(p_{1}, p_{2}, \ldots, p_{d+2}\right)$ included in the diagram of $T_{\text {aux }}$,

- $T^{\prime}$ is a minor of $T_{\text {aux }}$ corresponding to the other line $l^{\prime}=\left(p_{1}^{\prime}, p_{2}^{\prime}, \ldots, p_{d+2}^{\prime}\right)$ included in the diagram of $T_{\text {aux }}$,

- the unique point $p_{i}$ such that $p_{i}=p_{i}^{\prime}$ is (absolutely) removable in $l$, but is not (absolutely) removable in $l^{\prime}$.

- If $p_{1}=p_{1}^{\prime}$, then the edge $\left(T, T^{\prime}\right)$ is labeled by $1^{+}$. If the integer $i$ such that $p_{i}=p_{i}^{\prime}$ is not equal to 1 , then the edge $\left(T, T^{\prime}\right)$ is labeled by $i^{-}$if $p_{1}$ is covered by $p_{1}^{\prime}$, and labeled by $i^{+}$otherwise.

The only edges of the obstacle graph are those listed below (see Fig. 14):

- for each pair $(k, j)$ such that $1 \leq k<j \leq d+2$, the pair $\left(T_{k \mathrm{~L}}, T_{j \mathrm{~L}}\right)$ is an edge labeled by $k^{-}$(except the pair $\left(T_{1 \mathrm{~L}}, T_{(d+2) \mathrm{L}}\right)$ which is not an edge),

- for each pair $(k, j)$ such that $1<k<d+2$ and $d+3<j+k$, the pair $\left(T_{k \mathrm{~L}}, T_{j \mathrm{R}}\right)$ is an edge labeled by $k^{-}$,

- for each pair $(k, j)$ such that $1 \leq k<j \leq d+2$, the pair $\left(T_{k \mathrm{R}}, T_{j \mathrm{R}}\right)$ is an edge labeled by $k^{+}$(except the pair $\left(T_{1 \mathrm{R}}, T_{(d+2) \mathrm{R}}\right)$ which is not an edge),

- for each pair $(k, j)$ such that $1<k<d+2$ and $d+3<j+k$, the pair $\left(T_{k \mathrm{R}}, T_{j \mathrm{~L}}\right)$ is an edge labeled by $k^{+}$,

- for each integer $j$ such that $1 \leq j<d+2$, the pair $\left(T_{1}, T_{j \mathrm{~L}}\right)$ (we recall that $\left.T_{1}=T_{(d+2) \mathrm{L}}=T_{(d+2) \mathrm{R}}\right)$ is an edge labeled by $1^{+}$, and the pair $\left(T_{1}, T_{j \mathrm{R}}\right)$ is an edge labeled by $d+2^{-}$. 


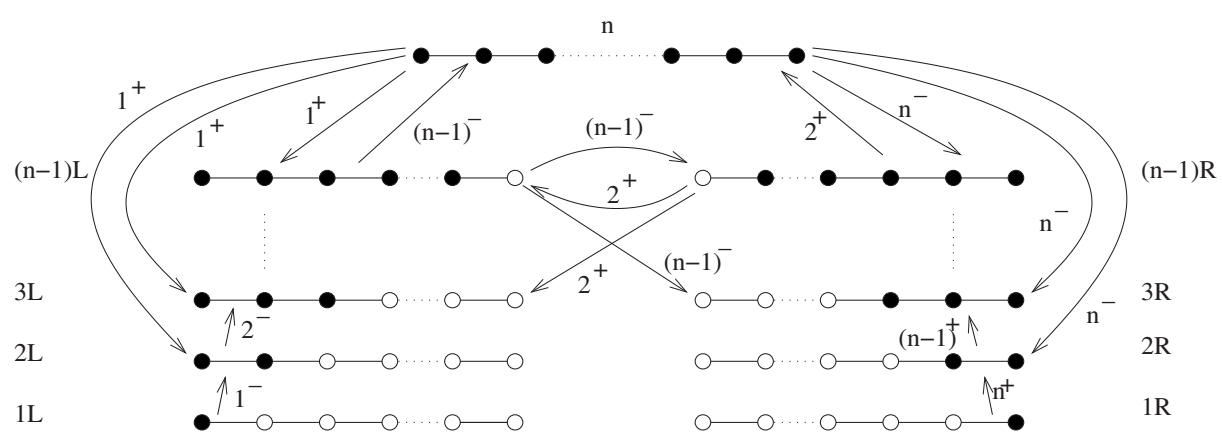

Fig. 14. The obstacle graph.

Lemma 5.4. We say that a directed cycle in the obstacle graph is equilibrated if the sequence of labels of its edges is such that, for each integer $i$ such that $1 \leq i \leq d+2$, the label $i^{+}$appears in the sequence if and only if $i^{-}$also appears. The obstacle graph has no (non-empty) equilibrated cycle.

Proof. Assume we try to find an equilibrated cycle. The vertices $T_{1 \mathrm{~L}}$ and $T_{1 \mathrm{R}}$ cannot be in the cycle, since they have no incoming edge. Then we remove them and all the edges adjacent to them, i.e. all the edges labeled by $1^{-}$and $(d+2)^{+}$. Because of the equilibration requirement for the cycle, we remove all the edges labeled by $1^{+}$or $(d+2)^{-}$.

We start again with vertices $T_{2 \mathrm{~L}}$ and $T_{2 \mathrm{R}}$. Since edges labeled by $1^{+}$and $(d+2)^{-}$have been removed, $T_{2 \mathrm{~L}}$ and $T_{2 \mathrm{R}}$ have no more incoming edges. Then they can be removed, as well as their outgoing edges, which are all the edges labeled by $2^{-}$and $(d+1)^{+}$. For equilibration requirements, one can also remove all edges labeled $2^{+}$and $(d+1)^{-}$.

With the procedure continuing, one can check that the whole graph will be deleted. Then there exists no equilibrated cycle.

\subsection{End of the Proof}

We now have the tools necessary to finish the proof of Theorem 5.1.

Proof of Theorem 5.1. As has been said before, the direct part is obvious. For the converse part, from the cluster reduction (Corollary 5.3) it suffices to prove that there exists a critical point.

For each line $l$, we say that a point $p$ is removable in $l$ if $p$ is removable for the pair $\left(T_{l}, T_{l}^{\prime}\right)$ of tilings of the unitary $(d+2)$-zonotope induced by the pair $\left(T, T^{\prime}\right)$ from the colorings of $l$.

If $T \neq T^{\prime}$, there exists a line $l_{0}$ of the diagram associated to $T$ containing a positive point $p_{0}$, which can be assumed to be removable in $l_{0}$. If $p_{0}$ is removable in $F_{p_{0}}$ for $\left(T_{p_{0}}, T_{p_{0}}^{\prime}\right)$, then we are done.

Otherwise, there exists a line $l_{1}$ passing through $p_{0}$, such that $p_{0}$ cannot be removed in $l_{1}$. In this case there necessarily exists another point $p_{1}$ which is removable in $l_{1}$. If $p_{1}$ is removable in $F_{p_{1}}$ for the pair $\left(T_{p_{1}}, T_{p_{1}}^{\prime}\right)$, then we are done. Otherwise, we can repeat the process. 
Assume that there is no point $p$ removable in $F_{p}$ for the pair $\left(T_{p}, T_{p}^{\prime}\right)$. With this hypothesis, the process can be infinitely repeated to construct a sequence $\left(l_{i}\right)_{i \geq 0}$ of lines. Thus there exists a finite subsequence $\left(l_{i}\right)_{i_{1} \leq i \leq i_{2}}$ which is a cycle, i.e. $l_{i_{1}}=l_{i_{2}}$.

This cycle is actually a cycle $C_{\text {line }}$ of the line graph. The main idea is the following: From the cycle $C_{\text {line }}$, one canonically obtains a cycle $C_{\text {obstacle }}$ of the obstacle graph, and from the mapping which associates to each line $l$ of the diagram, the (coloring given by the) tiling $T_{l}^{\prime}$ of the $(d+2)$-unitary zonotope. The edge labeling is preserved: if $\left(l, l^{\prime}\right)$ is an edge of $C_{\text {line }}$, then the edge $\left(T_{l}^{\prime}, T_{l^{\prime}}^{\prime}\right)$ obtained by mapping has the same label as $\left(l, l^{\prime}\right)$.

From the study of the obstacle graph, the cycle $C_{\text {obstacle }}$ is not equilibrated. One can assume without loss of generality, that the label $j^{+}$appears in the directed cycle, but the label $j^{-}$does not. This means that, following the cycle $C_{\text {line }}$, the $j$ th coordinate always changes in the same direction. This cannot arise. Thus the process must stop with a line $l_{i}$ and a point $p_{i}$ which can be removed.

\section{Structures for Lexicographic Sequences of Vectors}

We say that sequence $\left(v_{1}, v_{2}, \ldots, v_{D}\right)$ is lexicographic if for each line $l$, defined by a subsequence of $d+2$ vectors, the set of points of $l$ is ordered according to the lexicographic order of types.

Notice that the operations of contraction and deletion both preserve the lexicographic property.

We have seen that in codimension 2 , one can assume without loss of generality that the given sequence is lexicographic. This is not true in the general case.

Nevertheless, we can prove there exists a lexicographic sequence for any value of the parameters $d$ and $D$. It suffices to take a sequence $\left(v_{1}, v_{2}, \ldots, v_{D}\right)$ such that, for each subsequence $\left(v_{i_{1}}, v_{i_{2}}, \ldots, v_{i_{d}}\right)$, the determinant $\operatorname{det}\left(v_{i_{1}}, v_{i_{2}}, \ldots, v_{i_{d}}\right)$ is positive (see details in the Appendix). For example, this can be done by fixing an increasing sequence $\left(x_{1}, x_{2}, \ldots, x_{D}\right)$ of positive distinct reals numbers. We define $v_{i}$ by $v_{i}=\left(1, x_{i}, x_{i}^{2}, \ldots, x_{i}^{d-1}\right)$. Thus for each subsequence $\left(v_{i_{1}}, v_{i_{2}}, \ldots, v_{i_{d}}\right)$, the determinant $\operatorname{det}\left(v_{i_{1}}, v_{i_{2}}, \ldots, v_{i_{d}}\right)$ is a Vandermonde determinant, which is equal to $\Pi_{1 \leq i<j \leq d}\left(x_{j}-x_{i}\right)$, and, therefore, is positive. In the unitary case the order of the directed space of tilings is isomorphic to a higher Bruhat order [19].

Our main theorem about lexicographic sequences of vectors is stated below. It is a generalization of a theorem of Ziegler about higher Bruhat orders [19]. The idea is that even if Theorem 5.1 is false in the lexicographic case (Ziegler gives a counterexample in [19]), then there still remain many possibilities to pass from the lowest tiling to the highest one by a sequence of upwards flips.

Theorem 6.1. Let $T$ be a tiling of a zonotope constructed on a lexicographic sequence of vectors. There exists a sequence of downwards flips which can be done from $T$, satisfying the following properties:

- after the sequence is done, all points are white,

- the sequence can be decomposed into two parts, the first part only contains flips whose type contains $v_{D}$, and the second part only contains flips whose type does not contain $v_{D}$. 


\subsection{Arrow Properties in the Lexicographic Case}

In order to prove the theorem above, we first need the proposition below.

Proposition 6.2. Let $p$ and $p^{\prime}$ be two points linked by an arrow from $p$ to $p^{\prime}$, labeled by $D$. Assume that the last coordinate $D(p)$ of $p$ is lower than the last coordinate $D\left(p^{\prime}\right)$ of $p^{\prime}$. Then, for each pair $\left(p_{1}, p_{1}^{\prime}\right)$ such that there exists an arrow from $p_{1}$ to $p_{1}^{\prime}$ labeled by $D$, we have $D\left(p_{1}\right)<D\left(p_{1}^{\prime}\right)$.

Proof. For each pair $\left(q, q^{\prime}\right)$ of points, we denote by $\Delta\left(q, q^{\prime}\right)$ the number of coordinates which are null for $q$ and non-null for $q^{\prime}$. We use an induction on $\Delta\left(p, p_{1}\right)$. If $\Delta\left(p, p_{1}\right)=$ 0 , the result is just Proposition 4.5.

Otherwise, there exists a coordinate $i$ which is null for $p$ and not for $p_{1}$, and, in a symmetric way, a coordinate $j$ which is null for $p_{1}$ and not for $p$.

Let $p_{2}$ (respectively $p_{2}^{\prime}$ ) be the point whose $i$ th coordinate is null, whose $j$ th coordinate is the $j$ th coordinate of $p$ and whose other coordinates are equal to coordinates of $p_{1}$ (respectively $\left.p_{1}^{\prime}\right)$. We have $\Delta\left(p, p_{2}\right)=\Delta\left(p, p_{1}\right)-1$.

Let $l$ (respectively $l^{\prime}$ ) be the line passing through $p_{1}$ and $p_{2}$ (respectively $p_{1}^{\prime}$ and $p_{2}^{\prime}$ ). These two lines meet each other at a point $p_{0}$, whose last component is null. Since the sequence of vectors is acyclic, the point $p_{0}$ is an endpoint of lines $p$ and $p^{\prime}$. Applying the inversion property, we obtain that there exists an arrow from $p_{2}$ to $p_{2}^{\prime}$ labeled by $D$. Thus, by the induction hypothesis, we have $D\left(p_{2}\right)<D\left(p_{2}^{\prime}\right)$, i.e. $D\left(p_{1}\right)<D\left(p_{1}^{\prime}\right)$.

From the above proposition, up to symmetry, it can be assumed that, for each arrow labeled by $D$, the last coordinate of the origin point of the arrow is lower than the last coordinate of its tail point.

\subsection{Secondary Arrows}

We now introduce some other arrows, which are called secondary arrows, as follows: let $l=\left(p_{1}, p_{2}, \ldots, p_{D+2}\right)$ be a line. We assume that the point $p_{1}$ is the point of the lowest label in $l$. This point is called the directing point of $l$.

- If $p_{1}$ is black in $T$, then we have a secondary arrow from $p_{i}$ to $p_{i+1}$, for each integer $i$ such that $1 \leq i<D+2$.

- If $p_{1}$ is white in $T$, then we have a secondary arrow from $p_{i+1}$ to $p_{i}$, for each integer $i$ such that $1 \leq i<D+2$.

Secondary arrows are not labeled. They strongly depend on the tiling $T$, the opposite of the primary arrows introduced previously. They encode a sense for each line. Notice that the arrow constraint is still satisfied: there is no (secondary) arrow starting in a white point and finishing in a black point. A primary arrow links two points corresponding to tilings of the same zonotope, while a secondary arrow links two points corresponding to tilings of different zonotopes.

To avoid confusion, we say that the diagram with secondary arrows is the enriched diagram. 
Proposition 6.3. Let $T$ be a tiling. Each directed cycle of black points in the enriched diagram of $T$ is reduced to a single point.

Proof. The proof is done by induction on the size $s$ of the zonotope. The result is obvious for $s \leq d$ : there is no line in the diagram, thus the result comes directly from Proposition 4.5. This gives the initialization. Now, we assume that the size of the zonotope is at least $d+1$. We have two cases. In each of them we introduce a partition of points according to the two last de Bruijn zones.

(1) If $m_{D} \geq 2$, we define:

- $A_{0}=\left\{p \mid D(p) \leq m_{D}-2\right\}$,

- $A_{1}=\left\{p \mid D(p)=m_{D}-1\right\}$,

- $A_{2}=\left\{p \mid D(p)=m_{D}\right\}$.

If a black directed cycle remains in $A_{0} \cup A_{1}$, then it can be seen as a cycle of the diagram of $D_{\left\{v_{D}, m_{D}\right\}}(T)$ which gives the result by induction. Otherwise, let $p_{2}$ be a point in $A_{2}$ : there is no primary arrow from $p_{2}$ to any point $p$ outside of $A_{2}$, since such an arrow should be labeled by $D$, this would contradict Proposition 6.2. On the other hand, each line passing though $p_{2}$ has its directing point in $A_{0}$. Thus, if a black directed cycle has a point in $A_{2}$, then the cycle is necessarily contained in $A_{2}$ (since there is no possibility of having an arrow, in the cycle, starting in $A_{2}$ and finishing out of $A_{2}$ ). Thus this cycle can be seen as a cycle of the diagram of $D_{\left\{v_{D}, m_{D}-1\right\}}(T)$, which gives the result by induction.

(2) If $m_{D}=1$, we define:

- $A_{0}=\left\{p \mid(D-1)(p)<m_{D-1}\right.$ and $\left.D(p)=0\right\}$,

- $A_{1}=\left\{p \mid(D-1)(p)=m_{D-1}\right.$ and $\left.D(p)=0\right\}$,

- $A_{2}=\{p \mid(D-1)(p)=0$ and $D(p)=1\}$,

- $A_{2}^{\prime}=\left\{p \mid 0<(D-1)(p)<m_{D-1}\right.$ and $\left.D(p)=1\right\}$,

- $A_{3}=\left\{p \mid(D-1)(p)=m_{D-1}\right.$ and $\left.D(p)=1\right\}$.

Using Proposition 6.2 applied on $C_{\left\{v_{D, 1}\right\}}(T)$, it can be assumed that primary arrows linking a point of $A_{2}^{\prime}$ and a point in $A_{3}$ all finish in $A_{3}$ (there is a symmetric case when those arrows start in $A_{3}$, but the symmetric case can be treated in a symmetric way using the set $A_{3}^{\prime}=\{p \mid(D-1)(p)=1$ and $\left.D(p)=1\}\right)$.

Let $p_{2}$ be a point in $A_{2}$ : there exists exactly one line $l_{0}$ passing through $p_{2}$ and also containing (at least) a point of $A_{3}$. The directing point $p_{1}$ of $l_{0}$ is in $A_{1}$. All the points of $l_{0}$ are in $A_{3}$, except $p_{1}$ and $p_{2}$.

There exists exactly $m_{D-1}-1$ lines passing through $p_{2}$ and (at least) a point of $A_{2}^{\prime}$. The directing points of these lines are in $A_{0}$. The points of these lines are in $A_{2}^{\prime}$, except $p_{2}$ and the directing points. Moreover, from the inversion property, for each direction point $q$ of such a line, there exists a primary arrow from $p_{1}$ to $q$.

The other lines passing through $p_{2}$ are contained in $A_{0} \cup A_{2}$ and have their directing points in $A_{0}$.

On the other hand, if $p$ is a point in $A_{2}^{\prime} \cup A_{3}$, then each line passing through $p$ has its directing point in $A_{1}$ and its second point in $A_{2}$.

Thus, if a black directed cycle has a point in $A_{0} \cup A_{1}$, then it remains in $A_{0} \cup A_{1}$ (since there is no way for the cycle to pass from $A_{2} \cup A_{2}^{\prime} \cup A_{3}$ to $A_{0} \cup A_{1}$ ). Thus this cycle can be seen as a cycle of the diagram of $D_{\left\{v_{D}, 1\right\}}(T)$ which gives the result by induction. 

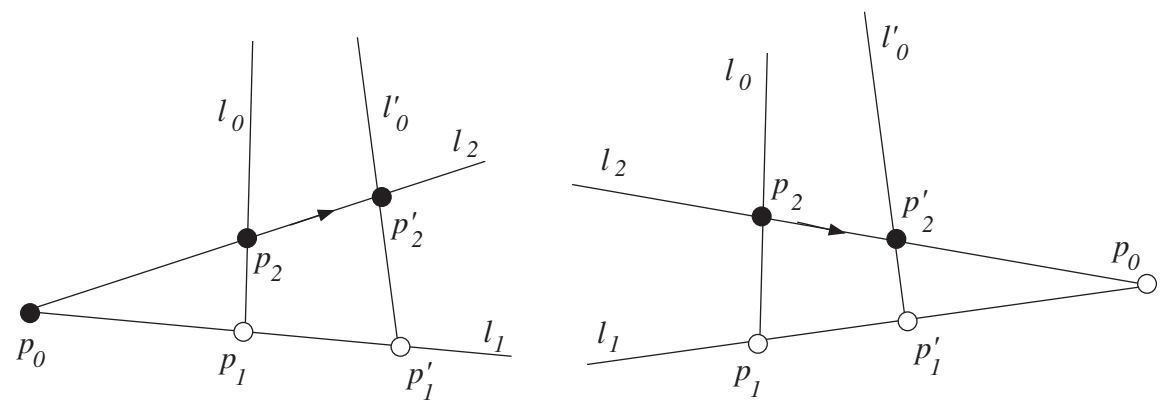

Fig. 15. The two possible cases, induced by the choice of the color of $p_{0}$. The color of $p_{1}^{\prime}$ is forced by colors of other points.

Now take a directed black cycle contained in $A_{2} \cup A_{2}^{\prime} \cup A_{3}$. Assume that there exists a point $p_{2}$ of $A_{2}$ in the cycle whose predecessor is an element of $A_{3}$. Let $l_{0}$ be the line passing by $p_{2}$ and its predecessor. The point directing point $p_{1}$ of $l_{0}$ is necessarily white, which implies that the directing point of each line passing by $p_{2}$ and having a non-empty intersection with $A_{2}^{\prime} \cup A_{3}$ is white. Let $p_{2}^{\prime}$ be the successor of $p_{2}$ in the cycle. From the color of the directing points above, $p_{2}^{\prime}$ cannot be in $A_{2}^{\prime} \cup A_{3}$. Thus $p_{2}^{\prime}$ is an element of $A_{2}$.

Let $l_{0}^{\prime}$ be the unique line passing by $p_{2}^{\prime}$ and containing points of $A_{3}$, and let $p_{1}^{\prime}$ be the directing point of $l_{0}^{\prime}$.

- If there exists a line $l_{2}$ passing through $p_{2}$ and $p_{2}^{\prime}$, then, by an elementary counting of the same coordinates, we see that there exists a line $l_{1}$ passing through $p_{1}$ and $p_{1}^{\prime}$. Moreover, $l_{1}$ and $l_{2}$ have a common directing point $p_{0}$ in $A_{0}$, and $p_{1}$ is between $p_{0}$ and $p_{1}^{\prime}$ if and only if $p_{2}$ is between $p_{0}$ and $p_{2}^{\prime}$ (see Fig. 15). The disposition above ensures that, in any case, the point $p_{1}^{\prime}$ is white (there are two cases according to the color of $p_{0}$ ).

- If there exists a primary arrow from $p_{2}$ to $p_{2}^{\prime}$, then (using the definition above) we directly get, thanks to the inversion property, that the point $p_{1}^{\prime}$ is white, since there is a primary arrow from $p_{1}$ to $p_{1}^{\prime}$.

In any case, we can repeat the argument to prove that the successor of $p_{2}^{\prime}$ is in $A_{2}$, and so on by induction. Thus the following points of the cycle are all in $A_{2}$, which is a contradiction. The study above proves that if the cycle has a point in $A_{3}$, then it is contained in $A_{3}$. Thus we have two alternatives, described below:

- either the cycle is contained in $A_{2} \cup A_{2}^{\prime}$, thus it is a cycle of the enriched diagram of $D_{\left\{v_{D-1}, m_{D-1}\right\}}(T)$ (notice that all the lines used by the cycle are contained in $A_{0} \cup A_{2} \cup A_{2}^{\prime}$, thus they are lines of $D_{\left\{v_{D-1}, m_{D-1}\right\}}(T)$ ),

- or the cycle is contained in $A_{3}$, thus it is a cycle of the enriched diagram of $C_{\left\{v_{D-1}, m_{D-1}\right\}}(T)$ (notice that all the lines used by the cycle are contained in $A_{1} \cup$ $A_{2} \cup A_{3}$, thus, for such a line, a line of $C_{\left\{v_{D-1}, m_{D-1}\right\}}(T)$ with the same directing point (in $A_{1}$ ) is obtained by removing the second (in the lexicographic order) point (in $\left.A_{2}\right)$ ).

In each alternative, we can apply an induction process to get the result. 


\subsection{End of the Proof}

We now have the tools to prove Theorem 6.1 easily.

Proof of Theorem 6.1. Let $\left(p, p^{\prime}\right)$ be a pair of points, on the same line $l$, such that the type of $p$ does not contain $v_{D}$ and the type of $p^{\prime}$ contains $v_{D}$. Assume that $p^{\prime}$ is black. The point $p$ is necessarily the directing point of $l$, thus if $p$ is white, then we have a secondary arrow from $p^{\prime}$ to $p$, and if $p$ is black, then we have a secondary arrow from $p$ to $p^{\prime}$. This yields that, in the enriched diagram, if a path of black points starts in a point whose type contains $v_{D}$, then all the types of points of this path contain $v_{D}$. Moreover, such a path is finite from Proposition 6.3. Therefore, if the enriched diagram of $T$ contains a black point whose type contains $v_{D}$, then the enriched diagram contains a black point whose type contains $v_{D}$ which is covered by no other black point. This last point can be turned into white to get another tiling. This operation can be repeated until there are no more black points whose type contains $v_{D}$.

Afterwards, one can select a black point which is not covered by another black point. This point can be turned into white to get another tiling. This operation can be repeated until there are no more black points.

\section{Consequences and Perspectives}

\subsection{Diameter, Connectivity and Homotopy}

From Theorems 5.1 and 6.1, we obtain a structure of a graded poset for the space of tilings. The maximal element is the fully black tiling, the minimal element is the fully white tiling. The rank of a tiling is the number of black points of its diagram. The height of the order is the sum $\sum_{1 \leq i_{1}<i_{2}<\cdots<i_{d+1} \leq d+2} m_{i_{1}} m_{i_{2}} \cdots m_{i_{d+1}}$. This height is also the diameter of the space of tilings. For $D-d=2$, the graded poset given is not a lattice in the general case. See a counterexample in Fig. 16. This is an important difference with the case $D-d=1$.

A natural way of improving the previous results is the study of the homotopy type of the space of tilings, which is called the general Baues problem [13] on polytopes.

\subsection{Uniform Random Sampling}

Consider the Markov process on tilings defined as follows: uniformly choose at random a point $p$ of the diagram, and a color $c$ (white or black). If the diagram obtained from $T$ giving the color $c$ to $p$ is the diagram of a tiling $T_{1}$ then replace $T$ by $T_{1}$.

Clearly, this process satisfies the hypothesis for ergodicity since the space of tilings is connected and there exist some loops in the process. Thus the probability distribution $p_{n}$ obtained after $n$ steps (starting from any distribution, for example, one can take for $p_{0}$ the Dirac distribution, concentrated on the wholly white tiling) converges to the uniform distribution. 


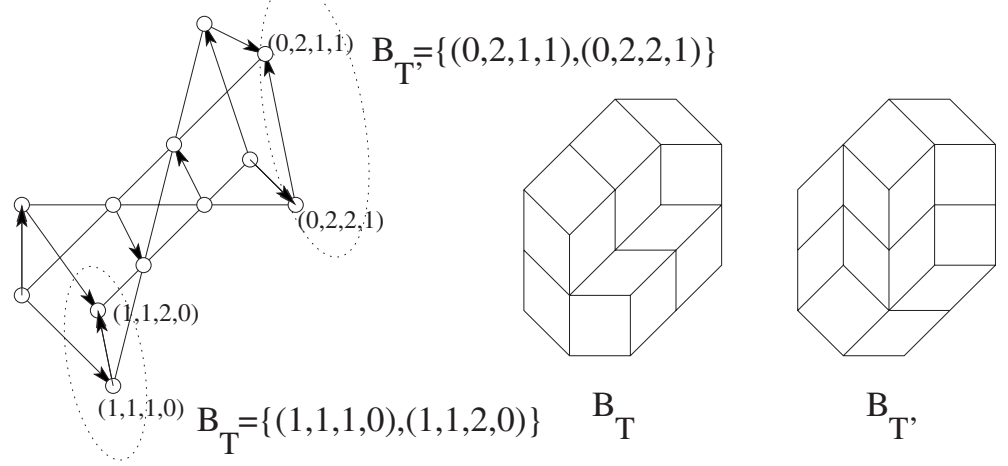

Fig. 16. The underlying diagram for two tilings having two incomparable suprema.

However, our result does not guarantee that the Markov process is rapidly mixing (even though it suggests a rather strong connectivity). The only positive fully proven positive results [12], [17] about rapid mixing are in the lozenge case $(c=1$ and $d=2)$. In an experiment by simulations, it has been obtained [6] that we also have rapid mixing in the octagonal case ( $c=2$ and $d=2$ ).

\subsection{Other Perspectives}

The representation used here gives an interesting approach for rhombic tilings. The associated diagrams give non-trivial results on the spaces of tilings. The connectivity result presented here looks really encouraging for further studies, and extensions of the flip graph may give more information on the sets of tilings, for example, flip distances between tilings. Moreover, the decomposition method seems to apply for more general polytopes.

\section{Appendix}

Proposition A.1. Let $\left(v_{1}, v_{2}, \ldots, v_{D}\right)$ be a sequence of vectors of dimension $d$ such that, for each subsequence $\left(v_{i_{1}}, v_{i_{2}}, \ldots, v_{i_{d}}\right)$, the determinant $\operatorname{det}\left(v_{i_{1}}, v_{i_{2}}, \ldots, v_{i_{d}}\right)$ is positive. This sequence is lexicographic.

Proof. For each subsequence $\left(v_{i_{1}}, v_{i_{2}}, \ldots, v_{i_{d}}, v_{i_{d+1}}, v_{i_{d+2}}\right)$, we study the tournament (introduced in 4.2.2) induced by $v_{i_{d+2}}$ on tiles on the lowest tiling $T$ of the unitary zonotope constructed on the sequence $\left(v_{i_{1}}, v_{i_{2}}, \ldots, v_{i_{d}}, v_{i_{d+1}}\right)$. There is no loss of generality in only studying the tournament induced by $v_{d+2}$ on the low tiling of the unitary zonotope $Z$ constructed on $\left(v_{1}, v_{2}, \ldots, v_{d}, v_{d+1}\right)$.

Each tile of $Z$ can be denoted by $t_{\bar{i}}$, where $i$ is the unique integer of $\{1,2, \ldots, d+1\}$ such that $v_{i}$ does not appear in the type of $t_{\bar{i}}$. 
For each pair $(i, j)$ of distinct integers, we denote by $\left\{f_{(\bar{i}, \bar{j}}^{+}, f_{\overline{(i, j})}^{-}\right\}$the pair of of facets of $t_{\bar{i}}$ such that $f_{\overline{(\bar{i}}, \bar{j})}^{-}+v_{j}=f_{\overline{(i}, \bar{j}}^{+}$. This common facet $t_{\bar{i}} \cap t_{\bar{j}}$ is denoted by $f_{\bar{i} \cap \bar{j}}$. Hence, either $f_{\overline{(i, \bar{j})}}^{+}=f_{\bar{i} \cap \bar{j}}$ or $f_{(\bar{i}, \bar{j})}^{-}=f_{\bar{i} \cap \bar{j}}$.

From the definition of the lowest tiling, $t_{\overline{d+1}}$ is in $T_{\left\{v_{d+1}, 1\right\}}^{-}$. Thus the facet $f_{\overline{d+1} \cap \bar{i}}$ is $f_{(\bar{i}, \overline{d+1})}^{-}$.

For any facet $f$ of $T$ and any vector $v$, we introduce the value $\operatorname{sign}(f, v)$ as the the sign (seen as an element of $\{-1,1\}$ for convenience) of the determinant $\operatorname{det}(S, v)$ where $S$ denotes the sequence of vectors of the type of $f$ (sorted according to the increasing indexes). Informally, $\operatorname{sign}(f, v)$ is a tool to know in what sense the vector $v$ passes through the facet $f$. For each vertex $v$ and each pair $(i, j)$, we have $\operatorname{sign}\left(f_{(\bar{i}, \bar{j})}^{+}, v\right)=\operatorname{sign}\left(f_{(\bar{i}, \bar{j})}^{-}, v\right)$, thus we can canonically define $\operatorname{sign}\left(f_{(\bar{i}, \bar{j})}, v\right)$.

From our hypothesis on determinants, we have $\operatorname{sign}\left(f, v_{d+1}\right)=\operatorname{sign}\left(f, v_{d+2}\right)=$ 1 for any facet of $t_{\overline{d+1}}$. Moreover, $\operatorname{sign}\left(f_{\overline{(d+1, \bar{i}},}, v_{i}\right)=(-1)^{d-i}$ since vectors of the corresponding determinant can be ordered in a lexicographic way by moving $v_{i}$ leftwards by a sequence of $d-i$ transpositions.

We now study the case when $d-i$ is odd (the case when $d-i$ is even is treated in a similar way). For $d-i$ odd, $\operatorname{sign}\left(f_{(\overline{d+1}, \bar{i})}, v_{i}\right)=-\operatorname{sign}\left(f_{(\overline{d+1}, \bar{i}}, v_{d+1}\right)$, which means that $v_{i}$ and $v_{d+1}$ pass through the face $f_{\overline{d+1} \cap \bar{i}}$ in opposite directions. Thus, since $f_{\overline{d+1} \cap \bar{i}}=f_{(\bar{i}, \overline{d+1})}^{-}$, we necessarily have $f_{\overline{d+1} \cap \bar{i}}=f_{\overline{(\overline{d+1}, \bar{i})}}^{-}$. For $i<d$, we also have $f_{\overline{d+1} \cap \overline{i+1}}$ is $f_{\overline{(\overline{d+1}}, \overline{i+1})}^{+}$, by the same argument (using that $\left.\operatorname{sign}\left(f_{(\overline{d+1}, \overline{i+1}}, v_{i+1}\right)=\operatorname{sign}\left(f_{(\overline{d+1}, \overline{i+1}}, v_{d+1}\right)\right)$.

The facet $f_{\bar{i} \cap \overline{i+1}}$ contains the $(d-2)$-face $f_{\overline{d+1} \cap \bar{i}} \cap f_{\overline{d+1} \cap \overline{i+1}}=f_{(\overline{d+1}, \bar{i})}^{-} \cap f_{(\overline{d+1}, \overline{i+1})}^{+}$. Thus $f_{\bar{i} \cap \overline{i+1}}$ is necessarily $f_{\overline{(i, \bar{i}+1})}^{+}=f_{\overline{(\overline{i+1}, \bar{i})}}^{-}$: Let $v$ be a vector in $f_{\bar{i} \cap \overline{i+1}}$; the vector $v$ is $f_{\bar{i}, \overline{i+1}}^{+}$, i.e. there exists a vector $v^{\prime}$ in $t_{\bar{i}}$ such that $v^{\prime}+v_{i+1}=v$. This enforces that $v$ is in $f_{(\bar{i}, \overline{i+1})}^{+}$(one proves in a symmetric way that $v$ is in $f_{\overline{(i+1}, \bar{i})}^{-}$).

Moreover, $\operatorname{sign}\left(f_{\overline{(i, \bar{i}+1})}, v_{i}\right)=\operatorname{sign}\left(f_{\overline{(i, \bar{i}+1})}, v_{i+1}\right)=(-1)^{d-i}$ since the corresponding determinant can be ordered in a lexicographic way by moving $v_{i}$ (or $v_{i+1}$ ) leftwards by a sequence of $d-i$ transpositions. Thus, $\operatorname{sign}\left(f_{\overline{(i,}, \bar{i}+1}, v_{i}\right)=-\operatorname{sign}\left(f_{\overline{(i,}, \overline{i+1}}, v_{d+2}\right)$ which yields that $v_{d+2}$ and $v_{i}$ pass through the facet $f_{\bar{i} \cap \overline{i+1}}$ in opposite directions. Thus the vector $v_{d+2}$ passes through $f_{\bar{i} \cap \overline{i+1}}$ from $t_{\overline{i+1}}$ to $t_{\bar{i}}$.

In other words, we have $t_{\overline{i+1}}<t_{\bar{i}}$ if the order is induced by $v_{d+2}$. One can prove the same result for $i$ even. Thus, we have $t_{\overline{d+1}}<t_{\bar{d}}<\cdots<t_{\overline{2}}<t_{\overline{1}}$. This yields that the sequence of flips successively labeled by $(\overline{1}, \overline{2}, \ldots, \overline{d+2})$ can be done, starting from the lowest tiling of the unitary zonotope induced by $\left(v_{i_{1}}, v_{i_{2}}, \ldots, v_{i_{d+2}}\right)$, which is the result.

\section{References}

1. A. Björner, M. Las Vergnas, B. Sturmfels, N. White and G. Ziegler, Oriented Matroids, second edition, Encyclopedia of Mathematics, vol. 46, Cambridge University Press, Cambridge, 1999.

2. F. Chavanon, Aspects combinatoires des pavages, Ph.D. thesis, ENS Lyon, 2004.

3. F. Chavanon and E. Rémila, Contractions of octagonal tilings with rhombic tiles, Tech. report RR2003-44, ENS Lyon, 2003. 
4. N.G. de Bruijn, Dualization of multigrids, J. Phys. France C(47) (1981), 3-9.

5. N. Destainville, Entropie configurationnelle des pavages aléatoires et des membranes dirigées, Ph.D. thesis, University Paris VI, 1997.

6. N. Destainville, R. Mosseri and F. Bailly, Fixed-boundary octagonal random tilings: a combinatorial approach, J. Statist. Phys. 102 (2001), 147-190.

7. S. Elnitsky, Rhombic tilings of polygons and classes of reduced words in Coxeter groups, J. Combin. Theory 77 (1997), 193-221.

8. S. Felsner, On the number of arrangements of pseudolines, Discrete Comput. Geom. 18 (1997), 257-267.

9. S. Felsner and H. Weil, A theorem on higher Bruhat orders, Discrete Comput. Geom. 23 (2003), 121-127.

10. S. Felsner and G. Ziegler, Zonotopes associated with higher Bruhat orders, Preprint.

11. R. Kenyon, Tiling a polygon with parallelograms, Algorithmica 9 (1993), 382-397.

12. M. Luby, D. Randall and A. Sinclair, Markov chain algorithms for planar lattice structures, SIAM J. Comput. 31 (2001), 167-192.

13. V. Reiner, The Generalized Baues Problem, 38, MSRI Book, Cambridge University Press, Cambridge, 1999.

14. J. Richter-Gebert and G. Ziegler, Zonotopal tilings and the Bohne-Dress theorem, Contemp. Math. 178 (1994), 211-232.

15. W.P. Thurston, Conway's tiling groups, Amer. Math. Monthly 97 (1990), 757-773.

16. M. Widom, R. Mosseri, N. Destainville and F. Bailly, Arctic octahedron in three-dimensional rhombus tilings and related integer solid partitions, J. Statist. Phys. 109 (2002), 945-965.

17. D.B. Wilson, Mixing times of lozenge tiling and card shuffling Markov chains, Ann. Appl. Probab. 14 (2004), 274-325.

18. Gr Ziegler, Lectures on Polytopes, Graduate Texts in Mathematics, Springer-Verlag, New York, 1995.

19. G. Ziegler, Higher Bruhat orders and cyclic hyperplane arrangements, Topology 32 (1992), 259-279.

Received June 9, 2004, and in revised form March 30, 2005, and April 22, 2005.

Online publication December 22, 2005. 